\title{
Dyes and Pigments
}

\section{Imaging and spectroscopic data combined to disclose the painting techniques and materials in the fifteenth century Leonardo atelier in Milan. \\ --Manuscript Draft--}

\begin{tabular}{|c|c|}
\hline Manuscript Number: & \\
\hline Article Type: & Research paper \\
\hline Keywords: & $\begin{array}{l}\text { Pigments in Renaissance paintings; Imaging Techniques; Portable X-ray } \\
\text { fluorescence; Fiber Optics Reflectance Spectroscopy; Raman, FT-MIR and FT-NIR } \\
\text { reflection spectroscopy; Hyperspectral imaging }\end{array}$ \\
\hline Corresponding Author: & $\begin{array}{l}\text { Letizia Bonizzoni } \\
\text { Università degli Studi di Milano, Dipartimento di Fisica } \\
\text { Milano, Italy }\end{array}$ \\
\hline First Author: & Anna Galli \\
\hline Order of Authors: & Anna Galli \\
\hline & Marco Gargano \\
\hline & Letizia Bonizzoni \\
\hline & Silvia Bruni \\
\hline & Matteo Interlenghi \\
\hline & Margherita Longoni \\
\hline & Arianna Passaretti \\
\hline & Michele Caccia \\
\hline & Christian Salvatore \\
\hline & Isabella Castiglioni \\
\hline & Marco Martini \\
\hline Abstract: & $\begin{array}{l}\text { The project "Leonardesque Artists beyond the Visible" was planned in the } 500 \text { th } \\
\text { anniversary of the death of Leonardo da Vinci, with the occasion of an exhibition held } \\
\text { in Milan in } 2019 \text {, where more than twenty works made by Leonardesque masters have } \\
\text { been put on display. Among them, five representative paintings made by his closest } \\
\text { pupils were selected for a comprehensive and multidisciplinary project. Portable non- } \\
\text { invasive imaging and spectroscopy techniques were applied to supply useful } \\
\text { information to scholars but also to the wider public: description of the material } \\
\text { composition of the pigments, of the preparation and of the binders, existence or } \\
\text { absence of underdrawing, and identification of the painters' technique and style. } \\
\text { Particular attention was paid also to the image processing techniques, mostly for } \\
\text { hyperspectral and radiographic data, to get the most from both innovative and } \\
\text { traditional techniques. }\end{array}$ \\
\hline Suggested Reviewers: & $\begin{array}{l}\text { Claudia Caliri } \\
\text { claudia.caliri@Ins.infn.it } \\
\text { Expert in XRF and application on } \mathrm{CH} \text {, especially paintings and detection of pigment }\end{array}$ \\
\hline & $\begin{array}{l}\text { Andreas Karydas } \\
\text { karydas@inp.demokritos.gr } \\
\text { Expert in the development of analytical methodologies, instrumentation and } \\
\text { interdisciplinary applications of X-ray fluorescence with emphasis on } \mathrm{CH} \text { areas }\end{array}$ \\
\hline & $\begin{array}{l}\text { Peter Vandanabeele } \\
\text { Peter.Vandenabeele@UGent.be } \\
\text { Expert in Raman, FT Raman and micro-Raman spectroscopy for the identification of } \\
\text { pigments }\end{array}$ \\
\hline
\end{tabular}


Milan, October 20, 2020

Dear Editor,

I wish to submit the paper "Imaging and spectroscopic data combined to disclose the painting techniques and materials in the fifteenth century Leonardo atelier in Milan.” by Galli, Gargano, Bonizzoni, Bruni, Interlenghi, Longoni, Passaretti, Caccia, Salvatore, Castiglioni, Martini, to be considered for publication in Dyes and Pigments.

The work we are submitting presents the analyses on a set of paintings by Lonardo da Vinci pupils. These masterpieces were studied with the occasion of an exhibition in Milan: multispectral analyses and chemical characterization of pictorial layers were performed in strict collaboration with art historians.

Portable non-invasive imaging and spectroscopy techniques were applied to supply useful information to scholars but also to the wider public: description of the material composition of the pigments, of the preparation and of the binders, existence or absence of underdrawings, and identification of the painters' technique and style. Particular attention was paid also to the image processing techniques, mostly for hyperspectral and radiographic data, to get the most from both innovative and traditional techniques.

Trusting the paper will be found suitable for publication in Dyes and Pigments, also on behalf of my co-authors, I thank you for your attention and look forward to your reply.

Yours faithfully

Letizia Bonizzoni 
Imaging and spectroscopic data combined to disclose the painting techniques and materials in the fifteenth century Leonardo atelier in Milan.

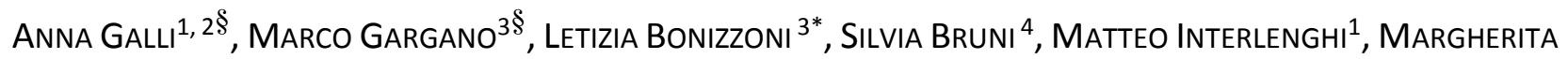
Longoni $^{4}$, Arianna Passaretti ${ }^{4}$, Michele Caccia ${ }^{2}$, Christian Salvatore ${ }^{6,7}$, Isabella Castiglioni ${ }^{5,1}$, Marco MARTINI ${ }^{2}$

${ }^{1}$ CNR-IBFM, via F.lli Cervi 93, Segrate (MI)

2 Dipartimento di Scienza dei Materiali, Università degli Studi di Milano-Bicocca, via R. Cozzi 55, Milano

${ }^{3}$ Dipartimento di Fisica Aldo Pontremoli, Università degli Studi di Milano, via Celoria 16, Milano

${ }^{4}$ Dipartimento di Chimica, via Golgi 19, Milano

${ }^{5}$ Dipartimento di Fisica Giuseppe Occhialini, Università degli Studi di Milano Bicocca, piazza della Scienza 3, Milano

${ }^{6}$ DeepTrace Technologies S.R.L., via Conservatorio 17, 20122, Milano

${ }^{7}$ Scuola Universitaria Superiore-IUSS Pavia, Piazza della Vittoria 15, Pavia

$\S$ these authors contributed equally to the work

*Corresponding author: Letizia Bonizzoni, Dipartimento di Fisica, via Celoria 16, 20133, Milano letizia.bonizzoni@mi.infn.it

\footnotetext{
Abstract

The project "Leonardesque Artists beyond the Visible" was planned in the $500^{\text {th }}$ anniversary of the death of Leonardo da Vinci, with the occasion of an exhibition held in Milan in 2019, where more than twenty works made by Leonardesque masters have been put on display. Among them, five representative paintings made by his closest pupils were selected for a comprehensive and multidisciplinary project. Portable non-invasive imaging and spectroscopy techniques were applied to supply useful information to scholars but also to the wider public: description of the material composition of the pigments, of the preparation and of the binders, existence or absence of underdrawing, and identification of the painters' technique and style. Particular attention was paid also to the image processing techniques, mostly for hyperspectral and radiographic data, to get the most from both innovative and traditional techniques.

Results highlighted for each author a peculiar painting technique showing hidden features such as pentimenti and the panel preparation methods, pigments, binders and varnishes.
} 


\section{Keywords}

Pigments in Renaissance paintings; Imaging Techniques; Portable X-ray fluorescence; Fiber Optics Reflectance Spectroscopy; Raman, FT-MIR and FT-NIR reflection spectroscopy; Hyperspectral imaging;

\section{INTRODUCTION}

2019 marked 500 years since the death of Leonardo da Vinci and in Milan, where he worked for 17 years, major exhibitions were dedicated to his life. One of them was held at the Poldi Pezzoli Museum (7 Nov, 2019 - 10 Feb, 2020): "Leonardo e la Madonna Litta" (Leonardo and the Litta Madonna), an exhibition focused on the temporary comeback of one of the most famous masterpieces from the State Hermitage Museum to the city where it was painted. Together with this masterwork, a selected group of twenty paintings and drawings borrowed from both public and private collections all around the world was on display. They were all painted by Leonardo and his closest pupils in the last two decades of the 15th century when the maestro lived in Milan at the court of Ludovico il Moro. In fact, during these Milanese years, Leonardo da Vinci was the Master of a large workshop: in the final decade of the fifteenth century, the great amount of commissions determined the need of a fair number of assistants. The atelier became a well-established model for important artistic production, involving painters such as Marco d'Oggiono, Giovanni Antonio Boltraffio and Francesco Napoletano, three of the oldest pupils present in Leonardo workshop since about 1490, creators of significant works that helped disseminating Leonardo's innovative style. Leonardo and the la Madonna Litta appealing event took place as a part of the national celebrations promoted and supported by the Italian Ministry of Culture, thanks to the patronage of the Regional Government of Lombardy, the Municipality of Milan, and the Bracco Foundation, which also funded the diagnostic project "Leonardesque Artists beyond the Visible", launched in this context. The project was planned in close relation with the exhibition itself, promoting relations between science and art, and was centered on a selection of paintings by some of the earliest pupils of Leonardo. In collaboration with art historians, conservators and restorers, it was possible to define a specific protocol of measurements and data analysis in order to achieve as large as possible information. A series of diagnostic analyses has been carried out under the coordination of the CNR Istituto di bioimmagini e Fisiologia Molecolare in cooperation with Università di Milano, Università di MilanoBicocca, and the innovative spin off of Scuola Universitaria Superiore (IUSS) from Pavia -DeepTrace Technologies S.R.L.. The Physics technologies applied to the study of paintings are widely regarded 
to give useful information to experts in the field, but also to the wider audience. Besides supplying the mere description of the pictorial material composition and the possible existence of preparatory drawing, if guided by specific questions, Physics technologies can help the identification of the painters' technique and style.

Although the consolidated use of limited materials, typical when dealing with Renaissance products of workshops, the rendering technique could vary greatly, from the wooden support to the preparation, imprimitura and pigment layers with different types of binding media and varnishes. Moreover, underdrawings and possible drawing transfer methods by means of cartoons can explain and reveal multiple information to cast light on the reconstruction of the story of a painting, especially in relation with the rest of the workshop production. Conveniently integrated with historical and documentary analysis, imaging and spectroscopic analyses have today the acknowledged - though not exclusive - role of favouring the understanding of processes and materials used for the execution of a pictorial work, by an interdisciplinary, non-destructive, noninvasive approach, and help the experts in responding to key questions, not only about the conservation and restoration of the work, but also about its interpretation. In spite of the growing interest around Leonardo, systematic studies through physical and chemical analyses are still not so many (Sarrazin et al., 2009; L. Keith and A. Roy, 1996; L. Keith et al., 2011; M. Spring et al., 2011; Bonizzoni et al., 2017; de Viguerie et al., 2009; de Viguerie et al., 2010; Elias and Cotte, 2008; Billinge et al., 2011; Galli et al., 2020; Gutman Rieppi et al., 2020; Caliri et al., 2020; Barberis et al., 2019). To this purpose, the project planned non-invasive scientific investigations, in accordance with a suitable protocol applied to all the paintings. The measurements and data analysis were chosen to be synergic and complementary and innovative data handling was applied, as detailed in the following sections. The experimental protocol was wider with respect to the previous ones applied in similar works, already quoted above, and allowed to completely characterize the works studied from a technical point of view. The art-historian experts were thus offered objective information, useful for establishing the best conservation practice and to support their evaluation and critical interpretation, as evident in the artwork records in the exhibition catalogue (Di Lorenzo and Marani, 2019). Nonetheless, the same analyses have been a perfect training for new technical and methodological developments in the Heritage Science, and a springboard for challenging developments. The research had also the benefit of being part of the exhibition itself, allowing visitors to discover the hidden features of the analysed paintings trough video contents expressly produced. 


\section{EXPERIMENTAL}

\subsection{The paintings}

The analyses presented in this paper were performed on five easel paintings during the conservative works prior to the exhibition "Leonardo e la Madonna Litta". The masterpieces selected for this project, all by Leonardo pupils, are part of Poldi Pezzoli Museum collection or were borrowed to the Museum on the occasion: The Virgin and Child (The Madonna of the Rose) by Giovanni Antonio Boltraffio (see Fig. 1Sa in supplementary materials), The Virgin feeding the Child by an unknown painter of the Lombard school (see Fig. 1Sb), and The Christ Child by Marco d'Oggiono are all held by the Poldi Pezzoli Museum in Milan (see Fig. 1Sc). The Virgin and Child (see Fig. 1Sd) by Francesco Galli, known as Francesco Napoletano, is held by the Brera Art Gallery in Milan, and The Virgin and Child (see Fig. 1Se) by unknown painter of the Lombard school is property of a large private collection.

\subsection{Methods}

All the paintings were studied in situ, either at the owner institution or, in case of restoration still in progress, in the restorer working space. The goal of the research was to give art historians and restorers as much information as possible, to highlight the painting technique, in term of underdrawing, panel preparation and reconstruction of the pictorial techniques. With this aim, besides the pigments used, we also tried to propose a stratigraphy sequences of pictorial layers. We performed different analytical techniques, by means of imaging investigations (Daffara and Fontana, 2011; Delaney et al., 2005; Legrand et al., 2014); and pointwise spectroscopic analyses for both material and stylistic characterization. We were thus able to hypothesize and deduce the kind of materials (pigments and binders) and how the painters used them in the studied masterworks. Moreover, although it was not possible to perform microscopic cross-section analyses, strictly 
avoided in the current project, the possible stratigraphy was deducted as the applied techniques,

exploiting different wavelength, have different penetration depth (Bonizzoni et al., 2008, 2007; Colombo et al., 2011; Gargano et al., 2020). In particular, ED-XRF (Energy Dispersive X-Ray Fluorescence), FORS (Fiber Optics Reflectance Spectroscopy), Raman and Fourier Transform Infrared (FTIR) analyses are all spot techniques that have already proved to be more effective when applied in synergy (Bonizzoni et al., 2015). Moreover, more in detail, UV induced fluorescence is linked to the outer surface of the pictorial layers while the IR radiation can reach deeper layers up to the preparation or imprimitura. Furthermore, the use of a multispectral IR reflectography approach allowed us to differentiate the pigment transparency and highlight specific features related to the drawing technique (Gargano et al., 2007).

On the other hand, $X$-rays penetrate deeply into the matter, usually crossing the complete pictorial stratigraphy. Moreover, outgoing characteristic X-ray fluorescence of medium-heavy elements can still pass the whole thickness, while relatively light elements' $\mathrm{X}$-rays (such as $\mathrm{Ca}$ and $\mathrm{K}$ ) have a higher probability to be absorbed, giving an incomplete information about the lowest layers, often in gypsum. Besides, FORS is a powerful technique for surface pigment characterization, but it undergoes difficulty in spectra interpretation arising from possibly altered surface (presence of dust, yellowing or old varnishes) or complex mixtures and dark shades (Bonizzoni et al., 2008; Gargano et al., 2012). Micro-Raman spectroscopy is a technique of choice for painting analysis (Centeno, 2016) because it allows a rapid, unambiguous and in situ non-invasive approach for the examined objects. Since Raman spectroscopy probes molecular and crystal lattice vibrations, it is sensitive to the composition, bonding, chemical environment, phase and crystalline structure of the examined material and therefore it is able to unambiguously identify the majority of the pigments; furthermore its spatial resolution allows to discriminate among different chemical components in pigment mixtures and the measurements are free from interferences, except for the fluorescence 
due to binding media and/or varnishes (Vandenabeele et al., 2000). Although the vibrational

information obtainable by Raman spectroscopy is similar to that obtained by infrared spectroscopy (IR), they are not identical but rather complementary due to the different selection rules governing vibrational Raman scattering and IR absorption (Vandenabeele et al., 2001). When applied in the laboratory, Fourier-transform infrared (FTIR) spectroscopy offers a fast analysis of micro-samples and is able to provide information on the nature of the organic and inorganic materials used by an artist. Its application in a non-invasive manner requires measurements to be performed in the reflection mode and, even if several examples of such application are reported in the literature, it still deserves further exploitation. Of particular interest is the different penetration depth that can be achieved in the medium-IR (MIR) and in the near-IR (NIR) regions. Indeed, NIR can penetrate more than MIR due to its higher energy, thus allowing to reach a greater depth than in MIR, where only a few micrometers are possible. Thus, in principle, by acquiring IR spectra from MIR to the longer-wavelength portion of NIR it is possible to probe a layered structure from the surface to underlying layers. In addition, thanks to the smaller absorption coefficients, good quality reflection spectra on undiluted materials can also be obtained. Finally, it is well known from the literature that NIR spectra are particular suitable for identifying organic binders in paintings (Vagnini et al., 2009). The association of the four chosen analytical techniques makes it possible to overcome the limit of each one and to detect all class of pigments (organic, inorganic, dyes...) and to recognize pigments having the same characterizing elements, but different chemical formula. The different penetration depths of the primary sources allow to infer, in the simplest cases, the stratigraphy without any sampling (Bonizzoni et al., 2008; Bruni et al., 2008; Miliani et al., 2010) Regarding imaging analyses, UV induced fluorescence investigates the very superficial pictorial layer while IR radiation can reach deeper layers up to the preparation or imprimitura. Furthermore, the use of a multispectral IR reflectography approach allows to differentiate the pigment transparency 
and highlights specific features related to the drawing technique (Gargano et al., 2007). X-ray radiography, given the nature of $\mathrm{x}$-rays to penetrate deeply into the matter, provides information about the spatial distribution of heavy materials in every layer of the painting and the support. However, the information is presented in a single 2D image, making it difficult to separate the information related to each layer. In order to detect the contribution of each layer, statistical calculations based on iterative subtractive method were applied through comparisons between the images of the pictorial layer (obtained in the visible spectrum) and the corresponding radiographic images. These statistical algorithms allowed to separate the radiopaque image of the pictorial layer from those belonging to lower layers (materials of preparation plus support).

Data analysis method applied to the hyperspectral $H(x, y, \lambda)$ results allowed to take into account the similarity of each hyper spectrum with the other ones by means of supervised classification procedures, such as Spectral Angle Mapper (SAM) (Comelli et al., 2011; Pelagotti et al., 2008; van der Weerd et al., 2003). The «end-member» have been chosen by referring to spectroscopic data; the result is a stack of images, $I(x, y, \alpha)$, where the value of each pixel represents the angle, $\alpha$, between the spectrum identified by $(x, y)$ and the end member.

\subsection{Experimental setups}

\subsubsection{High Resolution Multispectral Imaging}

The photographic system consisted of digital back Phase One IQ3, with 'Trichromatic' (100 MP) detector, able to detect radiation from 350 to $1000 \mathrm{~nm}$. For all the acquisitions a Schneider Kreuznach 120 mm LS f/4.0 Macro lens was used in combination with different filters and light sources to select the appropriate wavelength range for the investigation.

\subsubsection{Visible-reflected (Vis) image}


For the acquisition of this images, a UV-IR cut filter was used mounted on the lens of the device

described above. For the lighting were used 2 halogen lamps, maximum power $150 \mathrm{~W}$ with a condenser lens to optimize direct lighting of paintings and to reduce surface heating.

\subsubsection{Ultraviolet-induced (UVF) fluorescence}

Together with the UV-IR cut filter, a $418 \mathrm{~nm}$ long-pass filter was applied to the same device. For the lighting two 3 W 365 nm LED light were used in a completely darkened room.

\subsubsection{Near Infrared (NIR) reflectography $(850-1000 \mathrm{~nm})$}

Two $150 \mathrm{~W}$ incandescent lights with an $850 \mathrm{~nm}$ long pass filter mounted on the lens of the device were used.

2.3.5 Infrared False Color (IRFC) imaging: Vis and NIR image were combined using the green and red channel of the Vis image, shifted respectively to the blue and green channel of the IRFC image, and the NIR image was used shown in the red channel.

\subsubsection{ShortWave Infrared (SWIR) reflectography $(1000-1700 \mathrm{~nm})$}

This technique has been performed with a new portable scanning system prototype based on a spherical scanning system (Gargano et al., 2017): successfully used in previous important scientific campaing (Gargano et al., 2019) an InGaAs camera (Xenics Xeva- 1.7-640, 640×512 elements, spectral sensitivity: 1000-1700 nm) was mounted on a motorized head that allowed precise and small movements $(0.01 \circ$ resolution for the rotations and $0.1 \mathrm{~mm}$ for the linear stage). For all the multispectral acquired images the resolution was of $20 \mathrm{pixel} / \mathrm{mm}$.

\subsubsection{Images in visible light with optical microscope}

A digital optical microscope AM4013MZ with polarizing filter (5 MP, 220x, magnification) was used to obtain magnified images in the visible range.

\subsubsection{Hyperspectral imaging in the range $400-1000 \mathrm{~nm}$}


Hyperspectral imaging was performed using a Specim IQ spectral camera. It employs the push broom method (Hartley and Gupta, 1994) measurements of a scene made by scanning line-by-line across a spectral range of $400-1000 \mathrm{~nm}$, thereby capturing the spectral information for each line of the scene across that spectral range. Across that spectral range, there are 204 binnable bands, partially overlapping, at $7 \mathrm{~nm}$ resolution. It is able to produce a square image of $512 \times 512$ pixels, each $17.58 \mu \mathrm{m} \times 17.58 \mu \mathrm{m}$ in size.

For the lighting, a system of 2 halogen lamps, with maximum power $150 \mathrm{~W}$, condenser lens to optimise direct lighting of paintings and to reduce surface heating, was used.

\section{$\underline{\text { 2.3.9 X-ray radiography }}$}

X-ray radiographies were obtained by a portable radiographic device (Xrayconsult), 20-80 kV was used to perform X-ray radiographies. To get the most from this technique, images acquired were elaborated to improve the signal to noise ratio, techniques to augment contrast through the application of filters, segmentation of region of interests, and extraction of characteristics useful for comparisons were applied.

\subsubsection{X-ray fluorescence spectroscopy (XRF)}

Assing LITHOS 3000 portable spectrometer was used, with quasi monochromatic excitation at 17.4 $\mathrm{keV}$, obtained with a transmission $\mathrm{Zr}$ filter (100 $\mu \mathrm{m}$ thick) mounted on a Mo target $\mathrm{X}$-ray tube, giving a $4 \mathrm{~mm}$ radius of analysis on the samples. The system was equipped with a Peltier-cooled Si-PIN detector, area of circular measurement $(\mathrm{d}=5 \mathrm{~mm}) . \mathrm{X}$-tube typical working conditions were $25 \mathrm{kV}$ and $300 \mu \mathrm{A}$ and the measuring time was about $30-60 \mathrm{~s}$. The efficiency of the handheld spectrometer is low for elements with $Z<17$. For each studied painting, please refer to the Supplementary Materials Section for the location of analysed points. (Fig 1Sa-e).

\subsubsection{Fibre optic reflectance spectrophotometry (FORS)}


A portable Vis-NIR spectrophotometer (HR4000, Ocean Optics Dunedin, FL, USA) was used for FORS analysis, with a linear array, $360-1100 \mathrm{~nm}, 2.7 \mathrm{~nm}$ spectral resolution with a $45^{\circ} \mathrm{x}: 45^{\circ}$ geometry of measurement and a detection area of about $2 \mathrm{~mm}^{2}$. The spectrophotometer was connected to a tungsten halogen light source (D65, HL2000, Ocean Optics): light is transmitted through a quartz fiber optics bundle 1.5-meter-long (Ocean Opticts), composed by six fibers ( $400 \mu \mathrm{m}$ each), to collect reflected light around the single central illuminating fiber $(400 \mu \mathrm{m})$. The spectrometer was connected to a laptop and calibrated using white and black reflectance standards (Spectralon ${ }^{\circledR} 99 \%$ and dark trap). Visible-NIR reflectance spectrum from $380 \mathrm{~nm}$ to $1000 \mathrm{~nm}$ was recorded for each sample. For each studied painting, please refer to the Supplementary Materials Section for the location of analysed points. (Fig. 1Sa-e).

\subsubsection{Raman spectroscopy}

Micro-Raman analyses have been performed by a compact portable Raman spectrometer I-Raman Plus BW Tec, with fibre optic configuration. The probe has a flexible fibre coupling encased in a protective jacketing material that performs Rayleigh scatter rejection as high as 10 photons per billion.

It was fixed to a xyz stage for micrometric positioning and the latter placed on a tripod. A diode laser emitting at $785 \mathrm{~nm}$ was used; the power at the sample was about $5 \mathrm{~mW}$, the spectral range of our measurements was $200-3000 \mathrm{~cm}^{-1}$, while the spectra resolution was about $3-5 \mathrm{~cm}^{-1}$.

The TE Cooled Linear Array detector (2048 pixel; pixel size $14 \mu \mathrm{m} \times 200 \mu \mathrm{m}$ ) was cooled by means of a Peltier device.

All spectra were obtained as a sum of 20 accumulations with an exposure time of $4 \mathrm{~s}$ for each accumulation. Raman spectra were analysed as obtained and the identification of compounds was performed comparing the spectra recorded on the painting with the ones belonging to our personal database of Raman spectra or to libraries available in the literature (Bell et al., 1997; Burgio and 
Clark, 2001). The database was created recording the Raman spectra on pure pigments and on reference paint samples prepared ad hoc. For each studied painting, please refer to the Supplementary Materials Section for the location of analysed points. (Fig. 1Sa-e).

2.3.13 Fourier-transform infrared spectroscopy (FTIR) A portable FTIR spectrometer Alpha Bruker equipped with an accessory for reflection measurements was used to acquire spectra from 7000 to $400 \mathrm{~cm}^{-1}$. Each spectrum was recorded as sum of 200 scans, with $4-\mathrm{cm}^{-1}$ resolution. The diameter of the measurement spot was $6 \mathrm{~mm}$.

First-derivatives of the NIR spectra were calculated by using Grams AI (Thermo Fisher) software. The Savitsky-Golay algorithm was used, with polynomial degree 2 and number of points from 16 to 33, according to the noise level of the spectra.

2.3.14 Fluorescence spectroscopy Just on some red details of one of the paintings, namely the Virgin and Child by Francesco Galli, fluorescence spectra were acquired with visible excitation at $435 \mathrm{~nm}$. The proper excitation wavelength was obtained from the emission of a halogen source (maximum power $150 \mathrm{~W}$ ) by an interference filter. The radiation was focused on the measurement area by a 20x microscope objective and the emitted radiation was collected through the same objective. A dichroic filter, with $458-680 \mathrm{~nm}$ transmission range, was used to eliminate the reflected incident radiation. The spectrum was acquired by a Lot Oriel MS125 spectrometer equipped with a Peltiercooled Andor CCD detector. Both the source and the spectrometer were connected to the microprobe by fibre optics.

For each studied painting, please refer to the Supplementary Materials Section for the location of analysed points. (Fig. 1Sa-e).

\section{RESULTS AND DISCUSSION}

The various issues discussed in the following represent a detailed catalogue of the techniques and materials used for the paintings, including a comprehensive discussion of the panel, preparatory 
layers, pigments and binding media, obtained not only from the non-invasive analyses but also from the application of image-processing algorithms. The summary below is intended to draw out some particular points and comparisons, as well as more general observations on the group of paintings considered here; although we studied panels of the same workshop, we found typical and different methods of realizing the artworks, from the panel to the upper paint layers.

\subsection{Wood Panel}

All the paintings in this study are on panels and, in one case, it was possible to identify the wood species starting from radiography. It is important to highlight that, in many cases, the radiographic images are not clear due to thickness of the preparation or imprimitura and the wood thickness with respect to the pigment layer where the X-ray absorption is far less. To overcome this issue, all radiographic images were elaborated to extract important features from distinct layers that would not otherwise be visible through traditional methods. For The Christ Child panel, by Marco d'Oggiono, the radiographic image (fig. $1 \mathrm{~A}$ ) was elaborated with iterative calculations of statistical probability using the comparison between the visible image and the radiographic image, in order to separate the radiopaque image belonging to the Christ Child (fig. 1B) from the one belonging to the wooden support (fig. 1C). As a first step of the procedure, mathematical processes - such as gaussian filtering, aimed to reduce image noises, and co-registration - were applied for a correct overlap of the radiographic image and the visible image, a necessary step for making local comparisons between the information provided by the two images. Secondly, statistical algorithms based on Bayesian filters were applied to separate radiopaque signals belonging to the different layers of the painting. The algorithms implemented were specially developed for the elaboration of this peculiar panel's images by the start up DeepTrace Technologies. In figure 1A, we can note the use of two different kinds of wood for the upper-median portion and the lower portion of the panel. The wood of the upper-median portion, better extracted by the radiography elaborations (fig. $1 \mathrm{C}$ ), shows 
parallel fibres differently from the wood structure visible in the lower part, which is to be attributed to an intervention made before 1836 (Di Lorenzo and Marani, 2019), that added about $3 \mathrm{~cm}$ to the original project designed by the author.

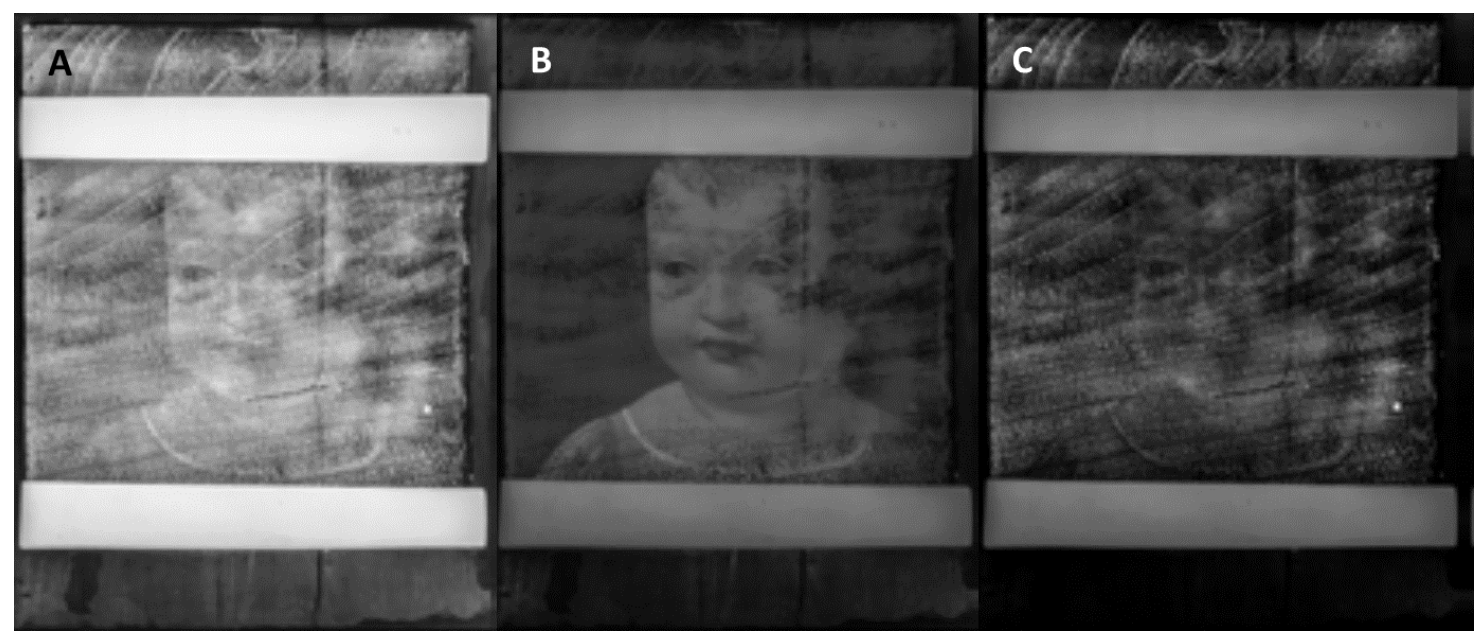

Fig 1: A) RX image; B) RX elaborated image highlighting the radiopaque layer of imprimitura; C) RX elaborated image highlighting the wood panel

From the literature, it is also possible to deduce information about The Virgin and Child (The Madonna of the Rose) by Giovanni Antonio Boltraffio. During the restoration carried out ten years ago (Beccaria, 2014), it was found to be painted on a single panel of walnut, 8 millimetres thick. It seems to have been a typical choice of painting support in Milan in the 1480 s and 90 s, when this wood was also widely used for furniture.

For the other three paintings, it was not possible to identify the type of wood used and the character of the grain as seen by eye on the reverse suggests that it might be oak for both the Virgin and Child paintings attributed to the Lombard school.

Even if Leonardo, in his Codex A of 1492, does not mention oak, nor poplar, as species used for paintings (but instead lists cypress, walnut, whitebeam and pear wood) oak is known to be the support he used for his French paintings while poplar was the most common wood used for panels during this period, generally speaking of Italian painters (M. Spring et al., 2011).

\subsection{Ground preparation and priming}


The panel support of The Virgin and Child by Boltraffio has been prepared with a layer of gesso mixed with a siccative oil. This preparation has been deduced from the comparison of XRF and FTIR experimental data. XRF detected in all the analysed points (Fig.1Sa) the presence of calcium among the minor elements; this is probably due to its presence in a deep layer, which leads to the absorption of its $k_{\alpha}$ and $k_{\beta}$ lines (respectively at $3.691 \mathrm{keV}$ and $4.013 \mathrm{keV}$ ). Moreover, in the NIR range (7500-4000 $\mathrm{cm}^{-1}$ ) for all the analysed areas (Fig.1Sa) it was possible to observe at 5140 and $6920 \mathrm{~cm}^{-1}$ the bands of gypsum $\mathrm{CaSO}_{4} \cdot 2 \mathrm{H}_{2} \mathrm{O}$ (Bacci et al., 1995), hypothetically associated with the preparation layer of the panel (Fig.2). It is interesting to note that in area 3 (Fig.1Sa), which on the basis of UV analysis appeared to have been restored (Fig.3), the gypsum signals are significantly stronger than those of the binder.

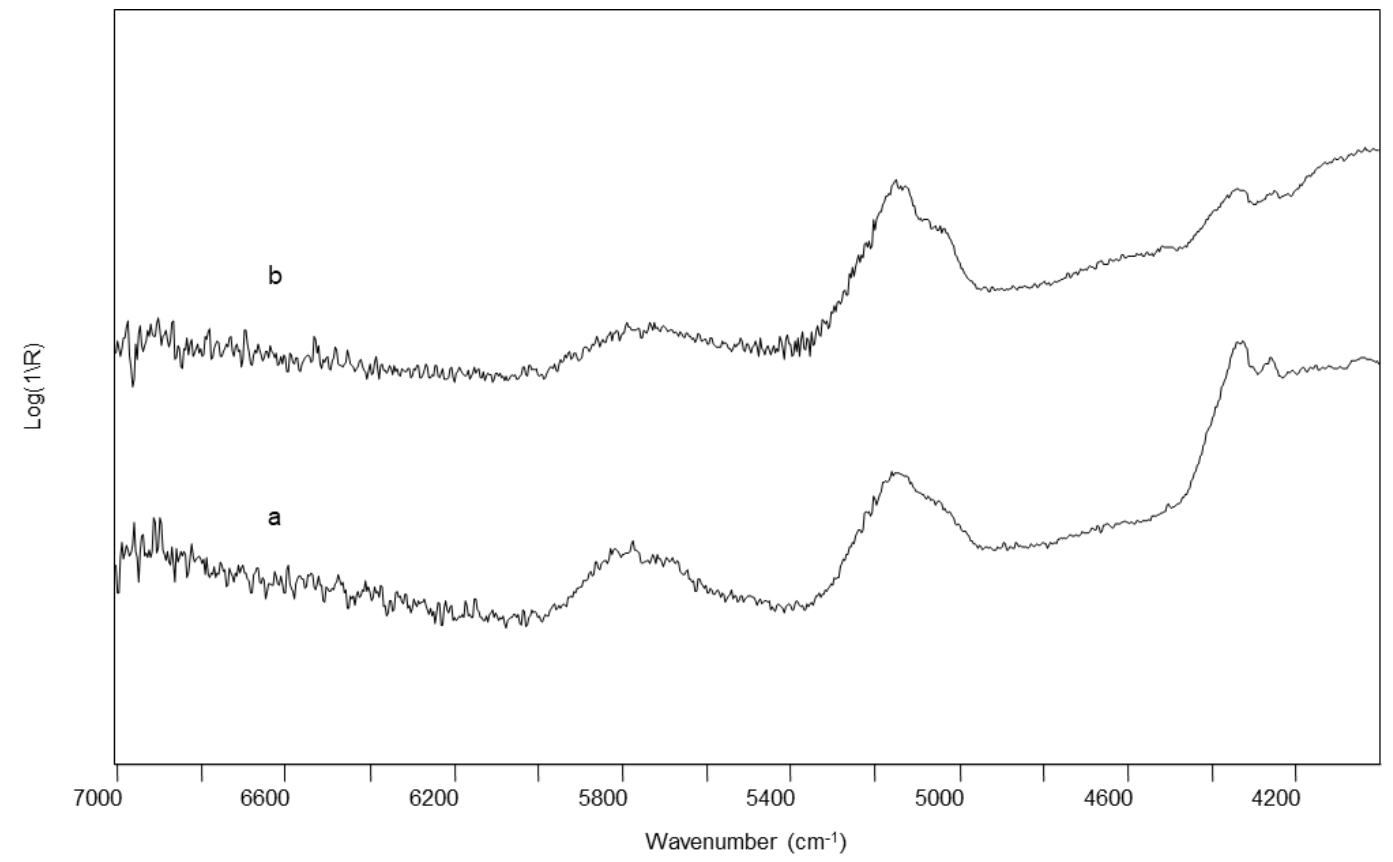

Fig. 2: FT-NIR reflection spectra of (a) area 2 and (b) area 3 of the painting "Virgin and Child" by G. Boltraffio

A priming consisting largely of lead based pigment (surely lead white, but with the presence of small amounts of red lead and lead-tin yellow (Beccaria, 2014) has been applied.

As already observed by restorer Carlotta Beccaria in her paper (Beccaria, 2014), this priming layer appears to have been applied with the painter's hands, mostly by the palm hands, to spread it onto 
the surface. In the $x$-ray image are in fact evident areas in which there is a non-uniform accumulation of priming layer (see fig.3b).

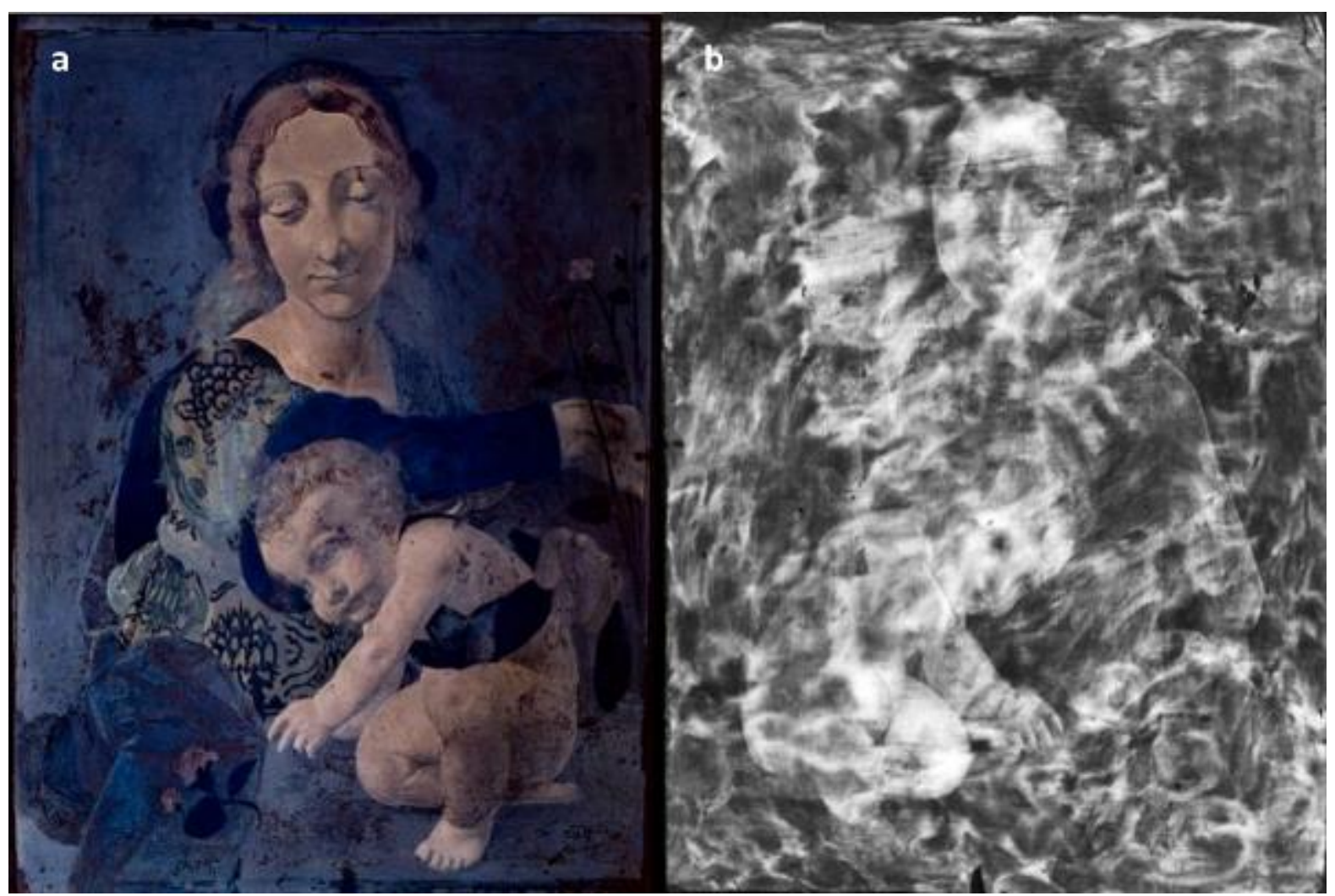

Fig. 3: panel a, on the left, UV image of the Virgin and Child by Boltraffio; panel b, on the right, RX of the Virgin and Child by Boltraffio.

The preparation of panel with gesso and the presence of a priming layer with the addition of earths/ochre have been also observed in the two Virgin and Child, both in the one by Napoletano and in the other by the unknown painter of the Lombard school.

Different are the cases of the Christ Child by d'Oggiono and the Virgin feeding the Child by an unknown painter of the Lombard school. For the latter panel, no evidence of preparation was found: neither XRF detects calcium, neither Raman nor FT-NIR spectroscopies individuate the typical bands of gypsum. Moreover, the priming seems to be layered with the same technique used by Boltraffio in Virgin of Rose: the X-ray image (fig.4) shows areas with different density distributed as leopard spots. 
Fig.4: RX of the Virgin feeding the Child

Also in the case of The Christ Child by d'Oggiono, no gypsum preparation was observed, except in the lower part of the painting that was in fact added later, as already pointed out, probably in the $19^{\text {th }}$ century, to enlarge its size.

\subsection{Underdrawing}

The heterogeneous procedures for realizing the considered artworks is confirmed by the study of underdrawings. Through the high-resolution reflectographic investigation, carried out using the InGaAs scanning system described above, it was in fact possible to detect the presence of 
underdrawings in all the investigated panels thank to the good penetration into the paint layers. In

Fig. 5 an overview of the five panels and their representative underdrawings is shown. As a basis for comparison the drawing of the hand was selected, except for The Christ Child panel were a detail of the Child face is shown. A fluid carbon based medium applied with brush was found for The Virgin feeding the Child (Fig 5-a) and in The Madonna of the Rose (Fig5-d) and Napoletano's painting (Fig. 5-e) where the drawing is particularly bold and sketched. For The Christ Child by Marco d'Oggiono (Fig. 5-c) a thin dry material trace was used to define the face elements, with the presence of a fine and detailed hatching for the shadows such as under the eyebrows and between the lips and the nose. In The Virgin and Child of the Lombard school (Fig. 5-b) a dry material was also used for the underdrawings. In all the paintings, the drawing appears to be made freehand: there are in fact slight modifications at this stage.

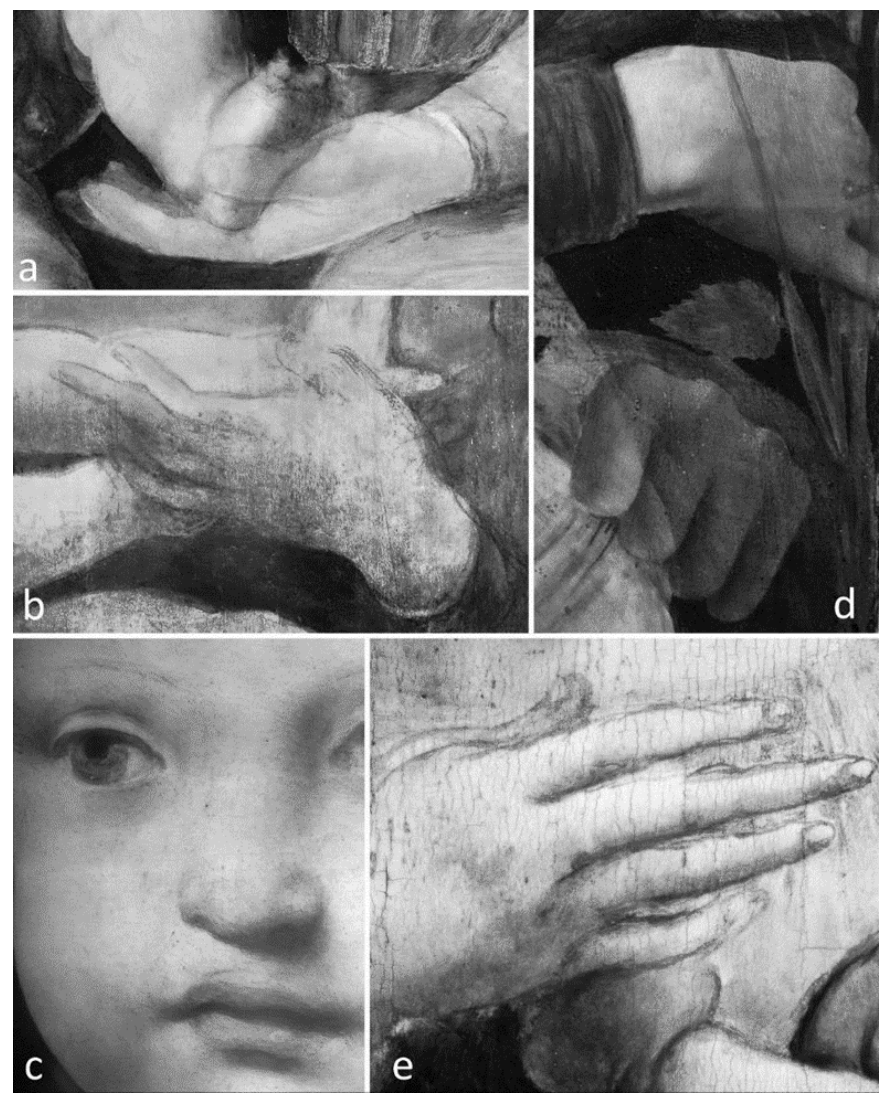

Fig. 5. Representation of the different types of underdrawing found in the five panels under investigation. a) A fluid carbon based medium applied with brush was found for the Virgin and child d) in the Madonna of the Rose and e) for the Napoletano's painting where the drawing is particularly bold and free. For the c) Christ Child by Marco d'Oggiono a 
thin dry material trace was used to define the face elements, with the presence of a fine and detailed hatching for the shadows. In the Virgin and Child of the Lombard school b) a dry material was also used for the underdrawing.

No evident traces of drawing transfer from possible cartoons were found except for The Virgin and Child of the Lombard school where limited and possible signs of pouncing were found in correspondence of the fingers (Fig. 6). Modification of the sketch are particularly interesting for two paintings: the Lombard school and the Napoletano panels. For The Virgin feeding the Child panel, several changes to the last version were made: (see Fig. $2 \mathrm{~S}$ in supplementary materials) for the Child, a shift of the fingers of the hand on the breast is present (Fig. 7-a), for the Virgin, a different position of one of her hands (Fig. 7-c). Comparing these two details acquired in the 1000-1700 nm range, it is noteworthy the evidence of two types of drawing materials, a fluid diluted ink applied with brush for the hand of the Child and a dry trace for the hand of the Virgin. It is interesting to note a detailed drawing of the head of the Virgin even under the veil that finally covers it (Fig. 7-b). The most important and radical modifications were however carried out on the background, which originally were realized as a vertical and rock-like landscaping, visible also beneath the curtain, with a building that almost entirely covered the portion of the background on the left (Fig. 8). This finding explains the results already obtained in 1988 for a stratigraphical sampling in the curtain area, when a layering of vermillion over azurite was detected (Antonietta Gallone, 1988).

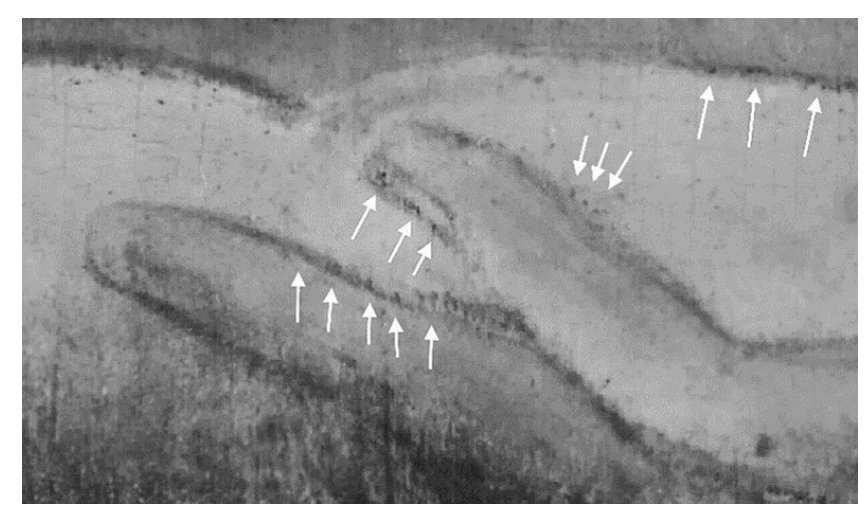

Fig. 6. Detail of the reflectogram for the Christ Child by Marco d'Oggiono: possible and limited traces of pouncing were found in correspondence of the fingers of the Virgin (white arrows). 
Fig. 7. Set of reflectographic details of the Virgin and Child of Lombard school. In these reflectograms it is possible to note modifications in the underdrawing $(a, c)$ with a repositioning of the fingers of the hands and $b$ ) a detailed drawing for the face and hair of the Virgin after having them covered with the application of the veil.

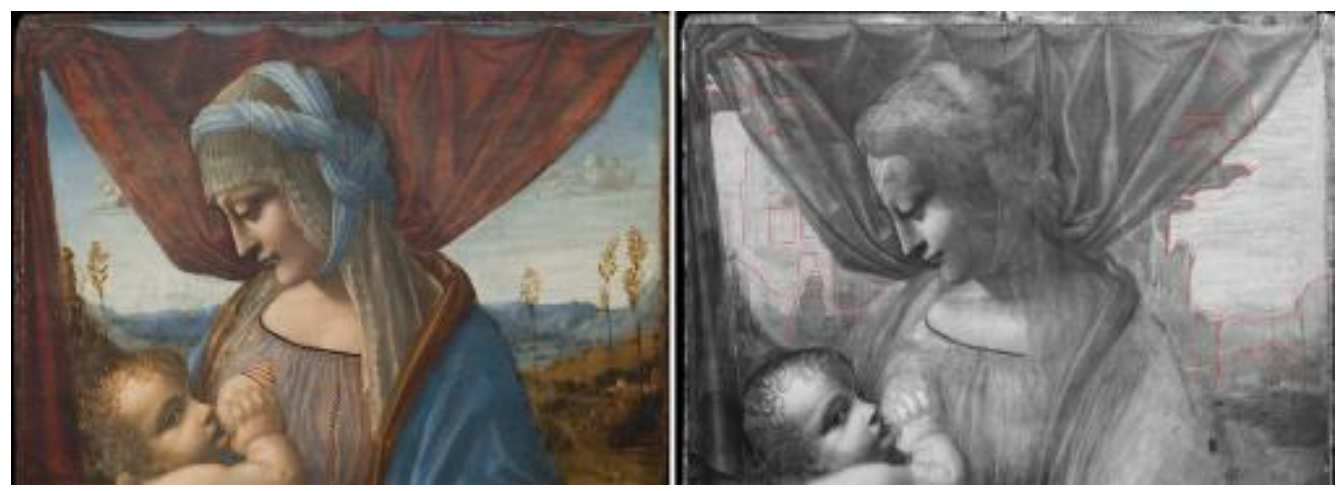

Fig.8. Virgin and Child of Lombard school, in the IR reflectogram is clearly showed that originally the background were realized as a vertical and rock-like landscaping, visible also beneath the curtain, with a building on the left that almost entirely covered the portion of the background.

For The Virgin and Child by Napoletano, beside the presence of a fluid freehand drawing with small changes in the paintings in many anatomical parts of the two figures, the most interesting finding is related to the presence of black fingerprints in various areas of the white background. In Fig. 9 is shown the IR reflectography of the neck of the Virgin where two distinct fingerprints have been firmly placed to add a dark background to the slope of the neckline painted subsequently. Other 
fingerprints were found also in other parts of the painting, again to darken, blend or connect some anatomical features.
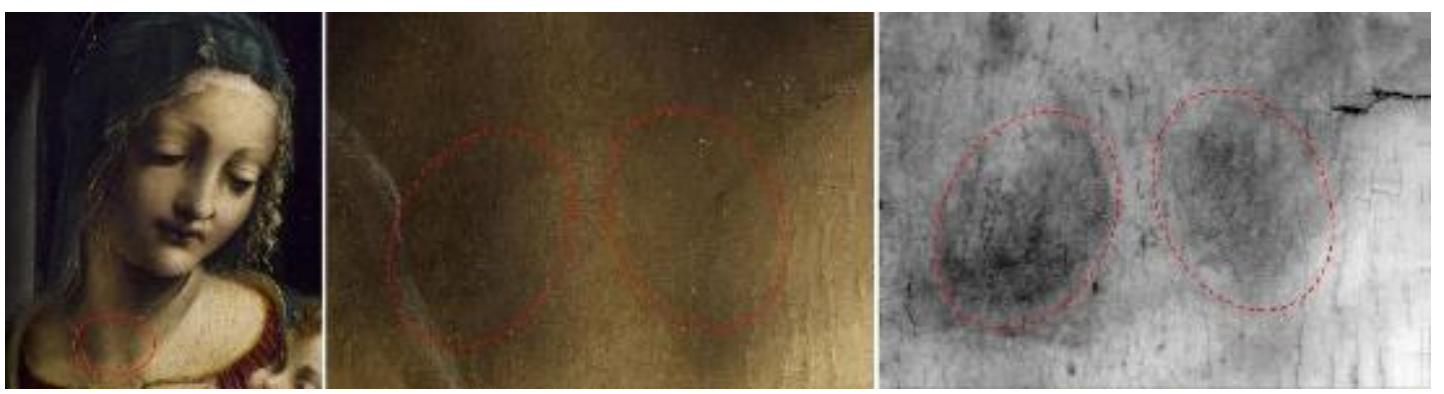

Fig. 9. IR reflectography of the neck of the Virigin: two distinct fingerprints have been firmly placed to add a dark background to the slope of the neckline painted subsequently.

\subsection{Paint layers}

The synergy between the imaging techniques and the spot spectroscopies gave us the possibility to infer the palette used by the five authors in the studied paintings with a complete non-invasive protocol. By studying the result of the UVF and microscope images, with the help of restorers, the areas to be analysed by spot techniques have been chosen. Regarding the painting materials, it is well known that in the Leonardo's workshop, exchange of drawings and ideas often occurred between pupils (M. Spring et al., 2011), so it is not surprising that the pigments used in all the five studied paintings are recurring. The chromatic effects were obtained with vermillion, lakes, earth in different shades (yellow, brown, red and golden), azurite and natural ultramarine, the blue from lapis lazuli stones, and lead based yellow. This section presents an overview of the detected pigments along with the speculated stratigraphic sequence of the pictorial layers for the most interesting cases; a complete summary of the results is reported in table 1.

Table 1: Overview of the pigment recognised in the considered paintings (for the collocation of measure points see fig. $1 \mathrm{~s}$ a-e).

\begin{tabular}{|c|c|c|c|}
\hline Points & Colour & \multicolumn{1}{|c|}{ Description } & Pigment from the synergy of spectroscopic techniques \\
\hline a & & \multicolumn{3}{|c|}{ Boltraffio, The Madonna of the Rose, Poldi Pezzoli Museum } \\
\hline $1,2,5,10,13$ & Skin tones & Virgin/Child & Yellow Ochre, Vermillion, White Lead \\
\hline 3 & Black & Background & Ochres in organic black \\
\hline 4,16 & Red & Flower & Vermillion \\
\hline 6 & \multirow{2}{*}{ Light blue } & Virgin dress & Azurite, Ultramarine \\
\cline { 3 - 4 } & & Virgin dress & Azurite, Ultramarine, Red Ochre (Hematite) \\
\hline 15 & &
\end{tabular}


Child belt/Virgin dress

Virgin and Child hair

Vermillion (organic pigments cannot be excluded)*

\begin{tabular}{|c|c|}
\hline 1,6 & Skin tones \\
\hline 2 & \multirow{2}{*}{ Dark red } \\
\hline 10,20 & \\
\hline 2 bis & \\
\hline
\end{tabular}

\section{Leaf} Yellow Ochre, Red Ochre Yellow Ochre, Red Ochre

Lombard School, The Virgin feeding the Child, Poldi Pezzoli Museum

\begin{tabular}{|c|c|}
\hline $4,5,11,14$ & \multirow{3}{*}{ Ligh blue } \\
\hline $8,16,17$ & \\
\hline 15 & \\
\hline 3 & \multirow{3}{*}{ Ochre } \\
\hline 7 & \\
\hline 13,13 bis & \\
\hline 9,19 & Light red \\
\hline 12 & Green \\
\hline 18 & \\
\hline
\end{tabular}

Child

Tenda

Cushion detail

Virgin dress and veil

$$
\text { Sky }
$$

Mountains

Windowsill

Child hair

Virgin dress edge

Tenda

Virgin veil

\begin{tabular}{c} 
Yellow Ochre, Vermillion, White Lead \\
Vermillion, Red Ochre with gold details \\
\hline Vermillion, Red Ochre
\end{tabular}

\begin{tabular}{|c|c|}
\hline $\mathbf{c}$ & \multicolumn{2}{|c|}{} \\
\cline { 1 - 1 } 1,3 & \\
\cline { 1 - 1 } & \\
\cline { 1 - 1 } &
\end{tabular}

\begin{tabular}{|c|c|}
\hline 6 & \multirow[t]{2}{*}{ Skin tones } \\
\hline 7 & \\
\hline 2 & Red \\
\hline 4 & \multirow{2}{*}{ Brown } \\
\hline 5 & \\
\hline $8,13,14$ & \multirow{2}{*}{ Blue } \\
\hline 9 & \\
\hline 10,15 & \multirow{2}{*}{$\begin{array}{l}\text { Dark } \\
\text { brown }\end{array}$} \\
\hline 11 & \\
\hline 12 & White \\
\hline d & \\
\hline
\end{tabular}

\begin{tabular}{|c|c|}
\hline Face & $\begin{array}{c}\text { Lead-tin yellow Type I, Yellow Ochre, Vermillion, White } \\
\text { Lead }\end{array}$ \\
\hline Neck & Yellow Ochre, Vermillion, White Lead \\
\hline
\end{tabular}

Shoulder (restoration)

Lead-tin yellow Type I, Yellow Ochre, Vermillion, White Lead

\begin{tabular}{|c|c|}
\hline Lips & \\
\hline Hair & \\
\hline Hair (restoration) & \\
\hline
\end{tabular}

Vermillion, Red Ochre

Dress (restoration)

Dress

Background

(restoration)

Background

Azurite, Ultramarine

Azurite, Ultramarine (gold traces)

Azurite, Ultramarine

Azurite, Ultramarine

Yellow Ochre

Yellow Ochre

Vermillion, Red Ochre

\begin{tabular}{|c|c|c|}
\hline $1,5,6,18$ & Brown & \\
\hline $2,3,10,16$ & Skin tones & \\
\hline
\end{tabular}

Francesco Galli (Napoletano), The Virgin and Child, Brera Museum

\begin{tabular}{|c|c|}
\hline $7,8,17$ & \multirow{2}{*}{ Yellow } \\
\hline 13 & \\
\hline 9 & \multirow{2}{*}{ Light blue } \\
\hline 12,15 & \\
\hline 11 & \multirow{2}{*}{ Red } \\
\hline 14 & \\
\hline $\mathbf{e}$ & \\
\hline
\end{tabular}

Virgin/ Child

Virgin halo, hair and

veil fringe

Virgin dress inside

Sky

Virgin dress

Virgin dress

Lombard School, The Virgin and Child, Palazzo Borromeo, Isola Bella

\begin{tabular}{|c|c|}
\hline 1 & \multirow{3}{*}{ Skin tones } \\
\hline 5 & \\
\hline $2,10,12$ & \\
\hline 3,11 & Blue \\
\hline 4 & Brown \\
\hline 6 & Ochre \\
\hline 7 & Ochre \\
\hline 8 & Light \\
\hline 9 & Blue \\
\hline
\end{tabular}

\begin{tabular}{|c|c|}
\hline Child leg & \\
\hline Child lips & \\
\hline Virgin & \\
\hline Virgin dress & \\
\hline Virgin dress & \\
\hline Child halo & \\
\hline Child hair & \\
\hline Mountains & \\
\hline Sky &
\end{tabular}

Lead-tin yellow Type I, Vermillion, White Lead

Lead-tin yellow Type I, Yellow Ochre, Vermillion, White Lead

Lead yellow, Vermillion, White Lead Azurite

Yellow Ochre, Azurite

Yellow Ochre (Azurite from the sky behind) 
Starting from these results, taking advantage of the hyperspectral imaging (HYS) on the whole

surface, the materials locally individuated by the comparison of data acquired by XRF, FORS, Raman and FTIR analyses have been detected and mapped in the paintings, with adequate data-processing methods.

It is well known that the HYS systems capture images at a large number of spectral bands and can identify materials with unique spectral features (Delaney et al., 2005; Legrand et al., 2014), which is a significant advantage compared with spectroscopic spot analyses. However, the spectral resolution of spectroscopic techniques is still much higher than that of HYS system (in our case: 2.7 $\mathrm{nm}$ spectral resolution for the FORS system versus $7 \mathrm{~nm}$ for the HSY instrument): for this reason, in the present work, the former have been used to support the processing and interpretation of HSY results.

In the following, for each painting, some peculiar aspects will be discussed, starting from The Virgin and Child by Boltraffio. In the last years, this panel has been submitted to different restoration works; during the 2011 intervention conducted by Carlotta Beccaria, it has been decided to remove some old instances of restoration that obscured the definition of the drapery of Virgin's cloak, making it less legible and flattening its form (Beccaria, 2014). Therefore, it has been interesting to study the original materials resurfaced: the blue drapery was originally painted applying a light under-layer containing lead white and azurite, covered by a layer of natural ultramarine. In fact, the blue areas investigated (points 6, and 15) show, based on the FORS spectra (fig.10a), the use of ultramarine. The presence of azurite, possibly mixed with white lead, is identifiable as an underlying stratum because of the high quantity of copper detected by XRF; the Raman spectroscopy performed on the areas 15 and 18 (see fig.1Sa) shows the band at $1314 \mathrm{~cm}^{-1}$ assigned to a twomagnon scattering which arise from the interaction of two magnons created on antiparallel close spin sites in $\alpha-\mathrm{Fe}_{2} \mathrm{O} 3$ (Hematite), see fig. 10b. Hematite perhaps belongs to a lower stratum, since 
the superficial layer is very much thinned, as the image at digital microscope acquired in the area 15 shows (Fig. 10c): there is a very thin-layered sequence in which the presence of a superficial layer of ultramarine and the presence of few red/brown grains are evident.

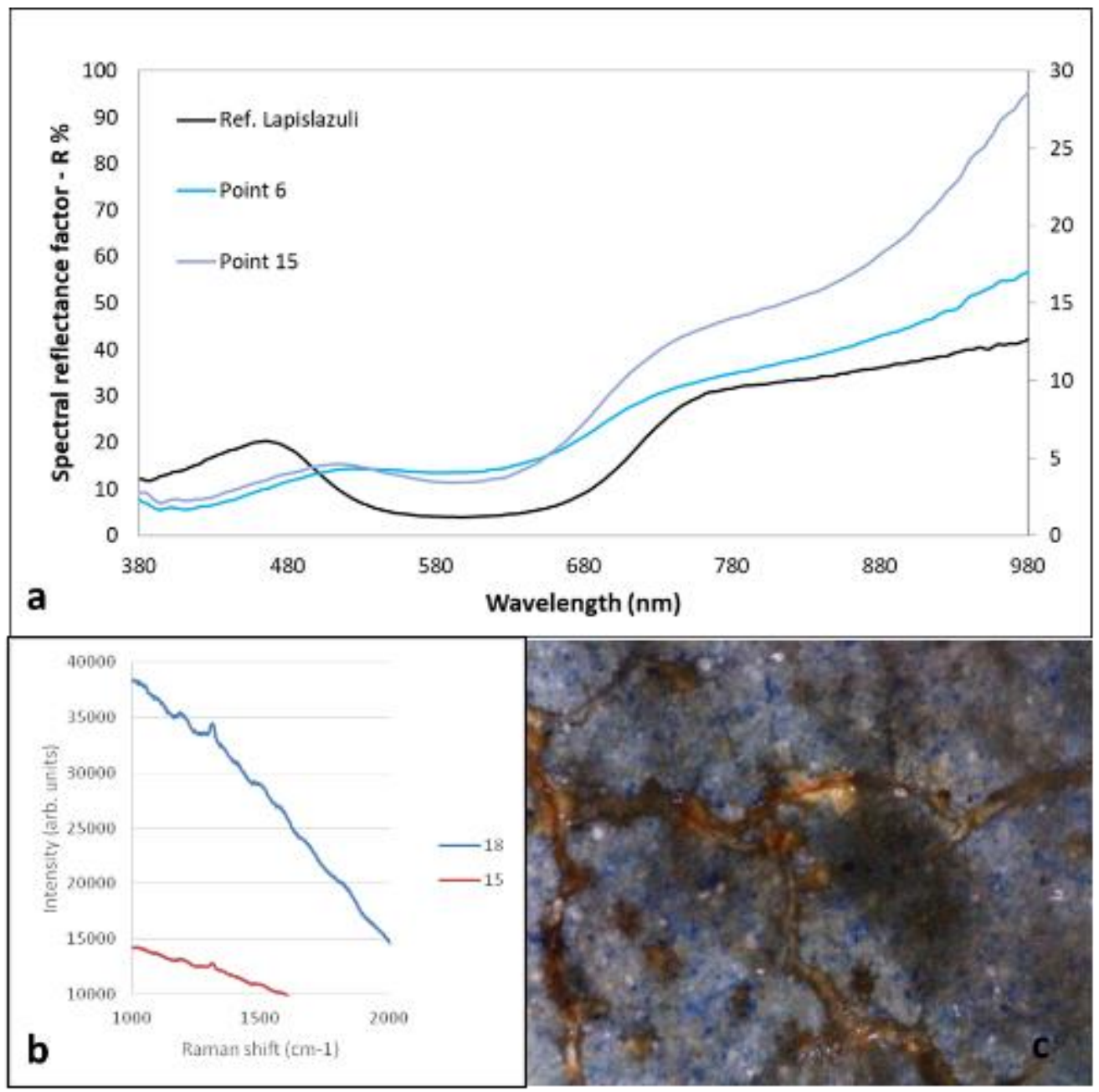

Fig.10: FORS (a) and Raman (b) spectra for the investigated blue areas on Boltraffio's Virgin and Child; corresponding digital microscope image is reported in (c).

It is precisely the presence of a precious pigment such as natural ultramarine, also spread in the landscape on the background and in the sky, that induce scholars to rethink the commission of The Virgin feeding the Child panel by the anonymous painter. Surprising is the use of this pigment for details negligible in the construction of the painting, such as the cushion's buttons (Fig. 11a); in fact, the Raman spectrum acquired on the 2_bis point (Fig.11b), shows the $\left(\mathrm{S}_{3}{ }^{-}\right)$symmetric stretching mode at $549 \mathrm{~cm}^{-1}$ ascribable to lapis lazuli (Hark and Clark, 2010). 

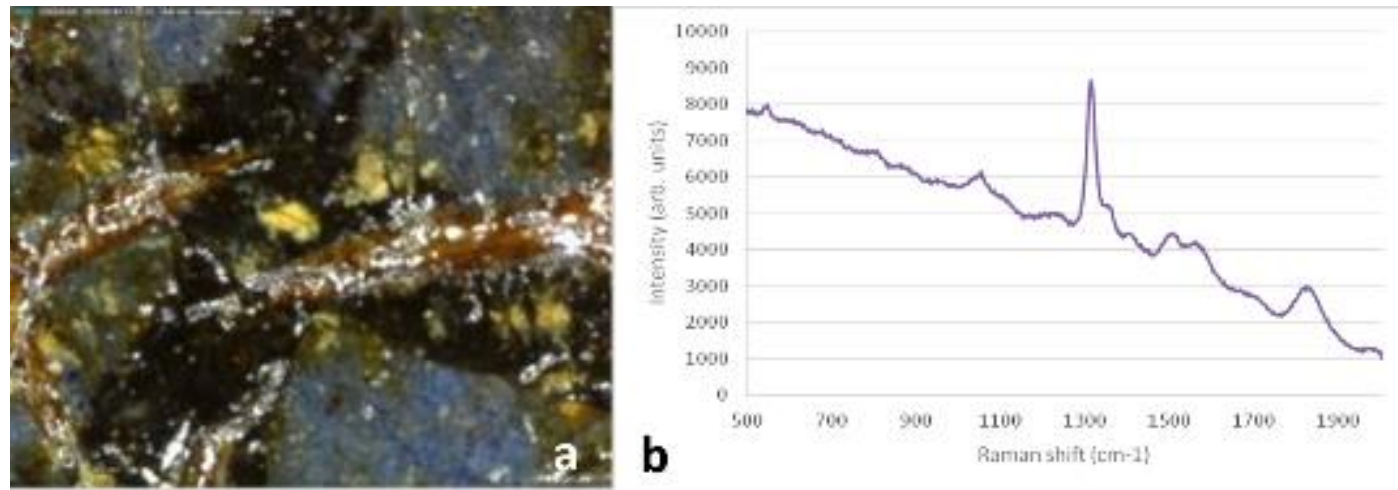

Fig.11: Blue detail for the button in the Virgin feeding the Child panel: digital miscroscope image (a) and Raman spectrum (b).

Moreover, the bands at $1314 \mathrm{~cm}^{-1}$ and $1355 \mathrm{~cm}^{-1}$ are present; they are two fluorescence emission bands resulting from an electronic mechanism activated by exposure of one of the associated minerals in this lapis lazuli sample to $785 \mathrm{~nm}$ radiation.

The band at $1,050 \mathrm{~cm}^{-1}$ is due to the presence of Lead white (hydrocerussite (lead(II) carbonate / lead(II) hydroxide).

The IRFC image helps in correctly mapping some of the pigments found on this painting with the above mentioned technique. The most interesting results are the characteristic purple color of the ultramarine and the yellow false color of vermilion used for the rose. (Fig. 3S in supplementary materials)

Also in his panel, Francesco Galli used precious materials; in particular, gold has been observed in many areas (see table 1). In the halo (point 7, fig. 1S), the veil on the hair (point 8 fig. 15 ), the dress (point 13 fig. 1S) and the golden fringe of the Virgin's veil (point 17 fig. 1S) it was detected together with copper, iron and lead by XRF. By comparing these data with those acquired with FORS and Raman spectroscopy, the presence of yellow ochre can be deduced. Moreover, in points 7 (fig. 12a), 8 (fig.12b) and 17 gold was used (fig. 12c), while at point 13 there was lead-tin yellow. 
These results are interesting because confirm the hypothesis given by the restorer of Brera Art

Gallery, Paola Borghese (Borghese, 2019), about the presence of a veil with golden fringes placed on the head of the Virgin, the veil the Child is playing with.

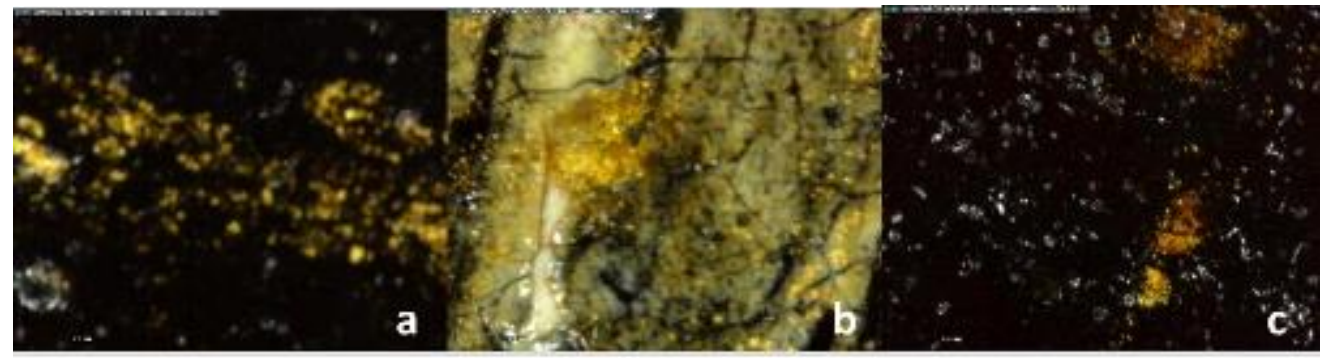

Fig. 12: Details of Francesco Galli panel, showing gold traces: a) point 7, the Virgin halo, b) point 8, the Virgin veil, c) point 17 , the Virgin dress.

For this panel, it was also possible to identify the red lake used for glazing with velatura technique the Virgin's dress. Red lakes were prepared from dyes both of vegetal (safflower, madder, and sappanwood) and animal (cochineal, kermes, and lac dye) origin, belonging to different molecular classes but especially to that of anthraquinones. In an extensive study about the painting materials used by the Milanese school at the end of the $15^{\text {th }}$ century (M. Spring et al., 2011), the use of both madder and kermes lakes was demonstrated by chromatographic analyses. In the present work, by measuring visible-excited optical fluorescence, the red lake in the painting by Francesco Galli could be recognised as deriving from a dye of animal origin, most probably kermes (Fig. 45 in supplementary materials). Also UVF images showed the presence of a red lake with its typical reddish-orangey fluorescence while in the IRFC image the azurite dress was highlighted. (Fig. 5S in supplementary materials)

The unknown master who painted The Virgin and Child used an unusual mixture of azurite and yellow ochre to paint the Virgin's dress, reaching a very peculiar chromatic effect. This mixture can be mapped by chosen as end-member the reflectance curve acquired on the dress (point 4 in fig.1S); 
as a result of the mapping procedure, performed on the basis of the SAM algorithm, we obtain the image shown in fig.13, a-c.

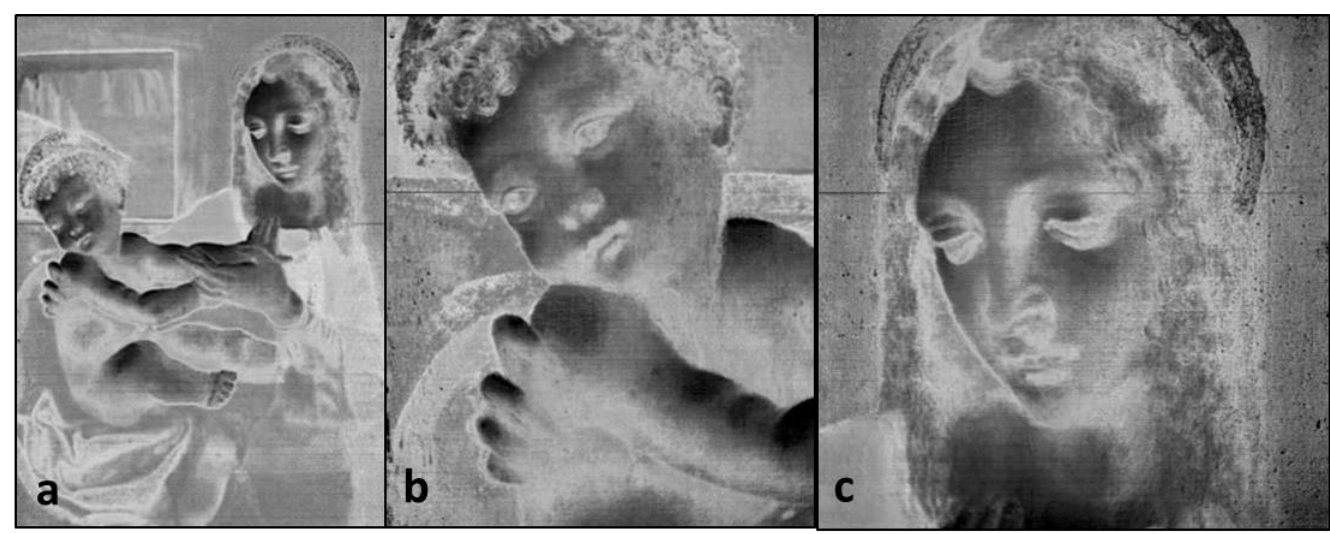

Fig. 13: Result of the mapping procedure for azurite and yellow ochre mixture in the unknown master panel, obtained on the bases of the SAM algorithm ( $a$, the whole panel, $b$, The Child detail, $c$ ) the virgin face). Lighter parts show a higher similarity, darker ones a higher difference.

One similarity map, $\mathrm{I}(\mathrm{x}, \mathrm{y}, 4)$, has been created by considering as reference the spectrum measured in point 4 (Fig. 1S). The reflectance curve shows a behaviour ascribable to yellow ochre (see the two band at $580 \mathrm{~nm}$ and $780 \mathrm{~nm}$ fig. 14) with the contribution of an additional pigment that, considering the specific reflectance rise in the wavelength range above $880 \mathrm{~nm}$, could be azurite.

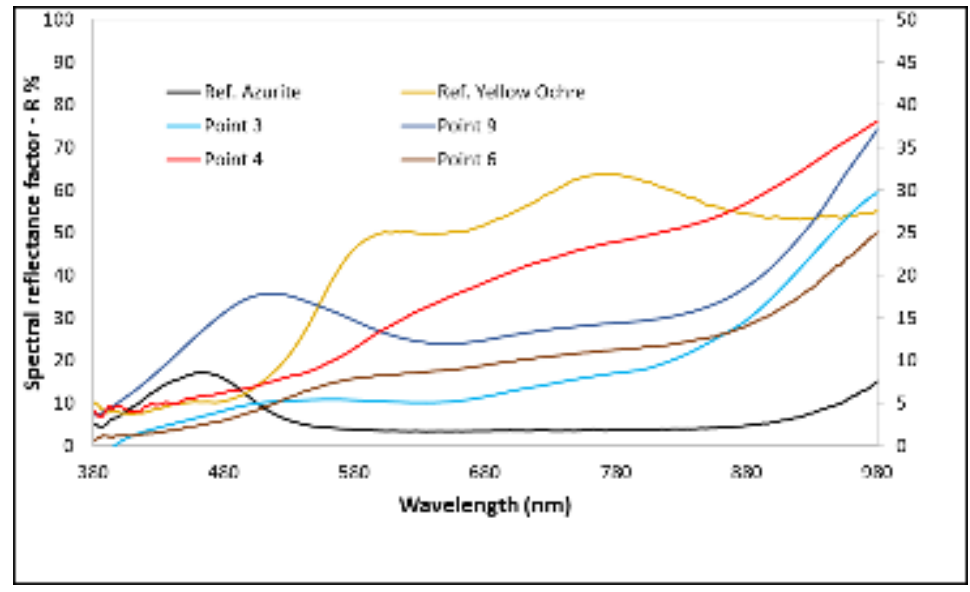

Fig.14: reflectance spectra for the Virgin and Child pane by an unknown painter (for the point location, see fig. 1S)

Moreover, analysing the results obtained in the same area by the comparison between XRF, Raman and FT-NIR data, we can deduce the presence of both azurite and yellow ochre. The presence of these two pigments is evident in the similarity map: in fact, the area of the Virgin's dress can be 
classified into two sub-regions with different spectral features: there are areas with a blue background with superimposed a yellow-brown painted layer. Moreover, there are other areas in which the mean reflectance spectrum of the blue background reveals spectral features similar to those of the blue pigment (identified as azurite) of Virgin's cloak and of mountain in the right part of the panel. The superimposed brown-yellowish layer reveals common features to yellow ochre that characterises the pigments mixture applied by the Master for the lips and the eyes of the Child (see fig.13b) and for the features and the shadows of Virgin's face (see fig. 13c). UVF image (Fig. 6S in supplementary materials) shows a damaged painting layers with many conservation interventions applied on the entire painting surface while the IRFC image helps in showing the distribution of azurite in the dress.

Particular is also how Marco d'Oggiono constructed the background in The Christ Child panel: in the visible range, the background appears very dark, but both FORS and XRF analyses confirm the presence of azurite, probably in addition to some organic brown/black pigments, as evident from the OM image (see fig.15) which highlights a blue hue diffuse with few inclusions of a brilliant light blue.

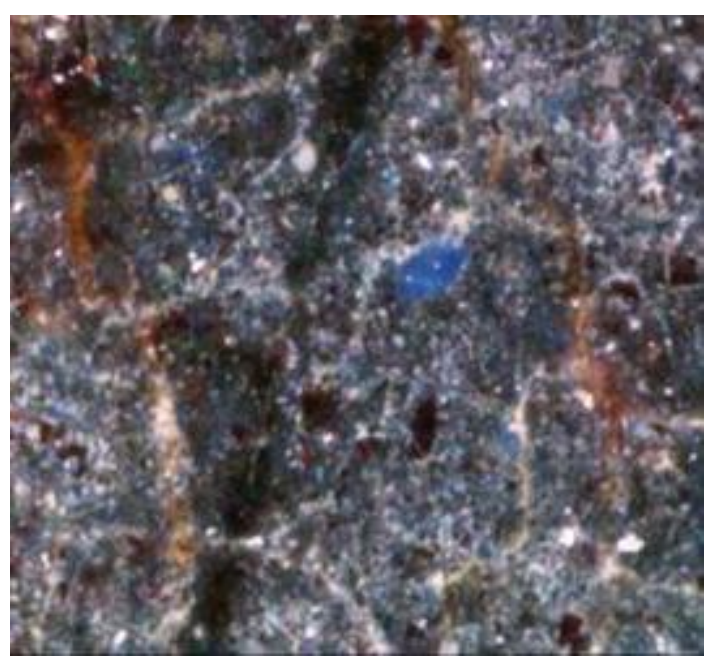

Fig.15: Digital microscope image for the dark background of the painting by Marco d'Oggiono. 
UVF analysis carried out during the conservation interventions, showed several integration areas positioned mainly in correspondence of the Child face and in the lower part of the panel. In this part there is in fact a junction that extends the height of the panel. IRFC showed the use of vermilion for the lips and highlights many retouching areas on the mantle of the Virgin.

Analyzing the painting under UV light, we mapped limited conservation interventions that appears as non-fluorescent areas. IRFC, as in the previous cases, highlights the Vermilion areas such as for the curtain and for the lapel and the purple false colour areas corresponding to the lapis lazuli used for the mantle of the Virgin, the background and the sky.

\subsection{Binders}

For all the examined areas of the five paintings of Leonardesque school, overtone and combination bands characteristic of siccative oils could be detected in the FT-NIR spectra at 4264, 4336, 5690 and $5784 \mathrm{~cm}^{-1}$ (Vagnini et al., 2009). Just in a detail of the hair of the Virgin in The Virgin and Child of unknown painter, the presence of gum arabic besides oil could be hypothesized, based on bands at 4020,4750 and $5175 \mathrm{~cm}^{-1}$ (Dong et al., 2017).

More challenging is instead the identification of the specific siccative oil used by the painters. To this aim, first-derivative of the spectra were calculated (Vagnini et al., 2009) and compared with those recorded for reference materials. In the already cited study about the painting practice of the Milanese Leonardo's pupils (M. Spring et al., 2011), analyses by gas chromatography-mass spectrometry confirmed that those artists usually employed an oil binder, supposed to be in most cases walnut oil. In the present study, reference samples were prepared by spreading fresh walnut and linseed oils on microscope glasses and then letting them dry, both naturally and by mild heating in an oven at $100{ }^{\circ} \mathrm{C}$ for 72 hours. The linseed oil sample was also submitted to light-induced ageing by a 6.5 hours-exposition to an Osram Powerstar lamp, with $150 \mathrm{~W}$ nominal power and emission between 390 and $600 \mathrm{~nm}$. In all cases, the two oils showed different first-derivative spectral 
patterns, especially regarding the component around $4400 \mathrm{~cm}^{-1}$, with slight shifts and changes in that the binder was indeed walnut oil, possibly heated (M. Spring et al., 2011), in the examined areas of The Virgin and Child by Galli (fig. 16e) and of The Virgin feeding the Child by unknown author. More variable results were obtained for the details considered in The Virgin and Child painted by Boltraffio. In fact, the first-derivative of the spectrum recorded on a detail from the black background resembled more closely that of linseed oil (fig. 16b), while for other areas the compresence of different oils, possibly also in superimposed layers, could not be ruled out. Interestingly, similar results were obtained by chromatographic analyses reported in the literature for samples from the same and also another painting by Boltraffio (Beccaria, 2014; M. Spring et al., 2011). Again, the use of walnut oil, but without completely excluding the presence of linseed oil, was suggested by the first-derivative NIR spectra also for The Virgin and Child of the Lombard school. A peculiar situation was encountered for The Christ Child by D'Oggiono, that could be analysed both before and after restoration. It should be recalled that the painting had been enlarged by a later addition of a wooden support, painted in imitation of the original work, and thus it had been probably glazed to make it homogeneous. Before removal of the later glaze, NIR spectra resembling that of walnut oil were obtained (fig. 16d), while, after cleaning, a greater similarity with linseed oil was observed (fig. 16c). Therefore, it can be supposed that linseed oil was the original binder for the Christ Child, while walnut oil was employed in the $19^{\text {th }}$-century restoration. 


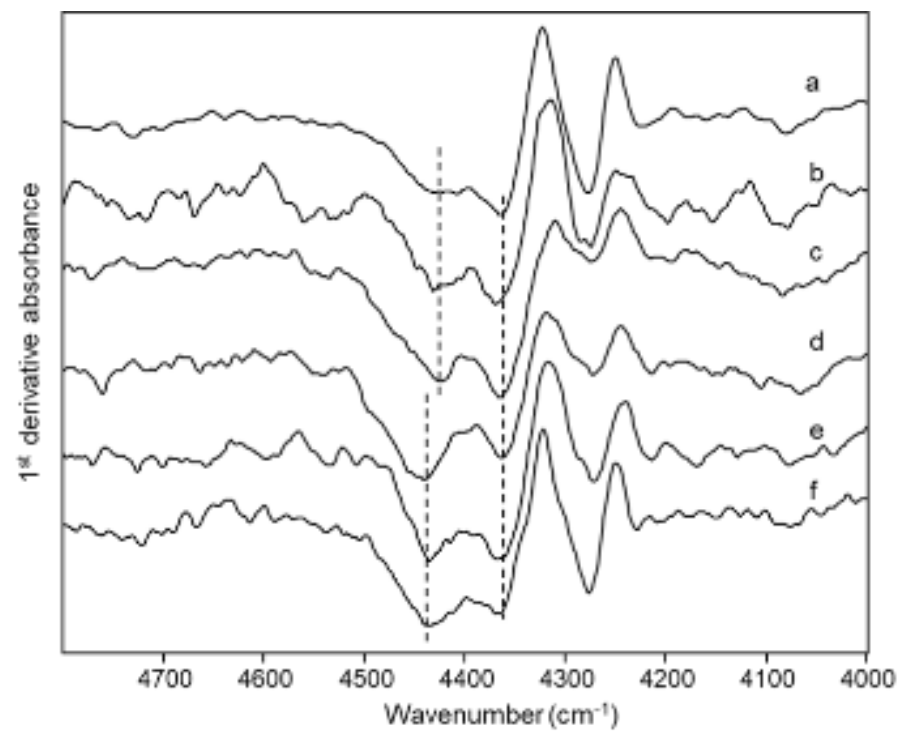

Fig. 16: First derivative of FT-NIR reflection spectra of: (a) linseed oil on a glass slide after 1-year ageing; (b) area 15 of the Virgin and Child by Boltraffio; (c) area 10 of the Christ Child by D'Oggiono (after cleaning); (d) area 3 of the Christ Child by D'Oggiono (before cleaning); (e) area 5 of the Virgin and Child by Galli; (f) walnut oil on a glass slide after heating at $100{ }^{\circ} \mathrm{C}$ for 72 hours. Dashed lines indicate the peaks whose positions and relative intensities were used to distinguish the two oils.

\section{CONCLUSIONS}

The project "Leonardeschi oltre il visibile" (Leonardesque Artists beyond the Visible), funded by Fondazione Bracco and whose main results are given in the present paper, is a good example of the synergy of scientific, conservation and humanistic studies since its planning. Working strictly in contact with art historians, restorers and curators of an important exhibition has enhanced the possibilities of technical analyses, giving focuses on the interesting puzzling themes. This is reflected also in the exhibition catalogue where the artwork records are linked to the results of scientific investigations, in the spirit of the studied Renaissance.

This same research also proved to be a good training for new technical and methodological developments, resulting in the possibility of a more complete, non-invasive, survey of both organic and inorganic materials. Also data analyses have been strengthening and developed to allow an easier and more effective handling of large sets of data from imaging techniques: in particular SAM (Spectral Angle Mapper) analyses applied to hyperspectral images and appropriate mathematical 
processes on Radiographic images opened a new way to the elaboration of data from Cultural Heritage artefacts.

The possible attribution of masterpieces is beyond the aims of the present research, but surely systematic studies through physical and chemical analyses can cast new light on the work of art historians.

\section{Acknowledgments}

The authors thank Brera Art Gallery and Poldi Pezzoli Museum for giving them the opportunity of carry out this research. They are grateful to Carlotta Beccaria and Paola Borghese for their trusting support. Giulia Guarnieri and Simone Caglio have collaborated in the data acquisition and processing. The project was funded by Bracco Foundation. 


\section{References}

Antonietta Gallone, 1988. Analisi di alcuni campioni di colore (Tehcnical Report on Madonna col Bambino).

Bacci, M., Baronti, S., Casini, A., Castagna, P., Linari, R., Orlando, A., Picollo, M., Radicati, B., 1995. Detection of Alteration Products in Artworks by Non-Destructive Spectroscopic Analysis. MRS Online Proc. Libr. Arch. 352. https://doi.org/10.1557/PROC-352-153

Barberis, E., Manfredi, M., Marengo, E., Zilberstein, G., Zilberstein, S., Kossolapov, A., Righetti, P.G., 2019. Leonardo's Donna Nuda unveiled. J. Proteomics 207, 103450. https://doi.org/10.1016/j.jprot.2019.103450

Beccaria, C., 2014. Some observations on the painting technique of Boltraffio's The Madonna and Child, in: Leonardo da Vinci's technical practice: Paintings, drawing and influence: Paintings, drawings and influence. M. Menu, Paris.

Bell, I.M., Clark, R.J.H., Gibbs, P.J., 1997. Raman spectroscopic library of natural and synthetic pigments (pre- $\approx 1850$ AD). Spectrochim. Acta. A. Mol. Biomol. Spectrosc. 53, 2159-2179. https://doi.org/10.1016/S1386-1425(97)00140-6

Billinge, R., Syson, L., Spring, M., 2011. Altered Angels: Two Panels from the Immaculate Conception Altarpiece once in San Francesco Grande, Milan. Natl. Gallery Tech. Bull. 32, 32-56.

Bonizzoni, L., Bruni, S., Galli, A., Gargano, M., Guglielmi, V., Ludwig, N., Lodi, L., Martini, M., 2015. Non-invasive in situ analytical techniques working in synergy: The application on graduals held in the Certosa di Pavia. Microchem. J. 126. https://doi.org/10.1016/j.microc.2015.12.001

Bonizzoni, L., Caglio, S., Galli, A., Poldi, G., 2008. A non invasive method to detect stratigraphy, thicknesses and pigment concentration of pictorial multilayers based on EDXRF and vis-RS: in situ applications. Appl. Phys. A 92, 203-210. https://doi.org/10.1007/s00339-008-4482-6

Bonizzoni, L., Galli, A., Poldi, G., Milazzo, M., 2007. In situ non-invasive EDXRF analysis to reconstruct stratigraphy and thickness of Renaissance pictorial multilayers. X-Ray Spectrom. 36, 55-61. https://doi.org/10.1002/xrs.930

Bonizzoni, L., Gargano, M., Ludwig, N., Martini, M., Galli, A., 2017. Looking for Common Fingerprints in Leonardo's Pupils Using Nondestructive Pigment Characterization. Appl. Spectrosc. 71, 1915-1926.

Borghese, P., 2019. La Madonna con Bambino di Francesco Napoletano alla Pinacoteca di Brera: Richiami leonardeschi e appunti in fase di conservazione. Kermes Riv. Restauro 91-98.

Bruni, S., Caglio, S., Guglielmi, V., Poldi, G., 2008. The joined use of n.i. spectroscopic analyses FTIR, Raman, visible reflectance spectrometry and EDXRF - to study drawings and illuminated manuscripts. Appl. Phys. A 92, 103-108. https://doi.org/10.1007/s00339-0084454-x

Burgio, L., Clark, R.J., 2001. Library of FT-Raman spectra of pigments, minerals, pigment media and varnishes, and supplement to existing library of Raman spectra of pigments with visible excitation. Spectrochim. Acta. A. Mol. Biomol. Spectrosc. 57, 1491-1521. https://doi.org/10.1016/s1386-1425(00)00495-9

Caliri, C., Bicchieri, M., Biocca, P., Romano, F.P., 2020. In situ macro X-Ray fluorescence scanning on a Leonardo da Vinci portrait. X-Ray Spectrom. n/a. https://doi.org/10.1002/xrs.3193

Centeno, S.A., 2016. Identification of artistic materials in paintings and drawings by Raman spectroscopy: some challenges and future outlook. J. Raman Spectrosc. 47, 9-15. https://doi.org/10.1002/jrs.4767 
Colombo, C., Bracci, S., Conti, C., Greco, M., Realini, M., 2011. Non-invasive approach in the study of polychrome terracotta sculptures: employment of the portable XRF to investigate complex stratigraphy. X-Ray Spectrom. 40, 273-279. https://doi.org/10.1002/xrs.1336

Comelli, D., Nevin, A., Valentini, G., Osticioli, I., Castellucci, E.M., Toniolo, L., Gulotta, D., Cubeddu, R., 2011. Insights into Masolino's wall paintings in Castiglione Olona: Advanced reflectance and fluorescence imaging analysis. J. Cult. Herit. 12, 11-18.

https://doi.org/10.1016/j.culher.2010.06.003

Daffara, C., Fontana, R., 2011. Multispectral Infrared Reflectography to Differentiate Features in Paintings. Microsc. Microanal. 17, 691-695. https://doi.org/10.1017/S1431927611000031

de Viguerie, L., Beck, L., Salomon, J., Pichon, L., Walter, P., 2009. Composition of Renaissance paint layers: simultaneous particle induced $\mathrm{X}$-ray emission and backscattering spectrometry. Anal. Chem. 81, 7960-7966. https://doi.org/10.1021/ac901141v

Delaney, J.K., Walmsley, E., Berrie, B.H., Fletcher, C.F., 2005. Multispectral Imaging of Paintings in the Infrared to Detect and Map Blue Pigments, in: Scientific Examination of Art: Modern Techniques in Conservation And Analysis. Natl Academy Pr, Washington, DC, p. 120.

de Viguerie, L., Walter, P., Laval, E., Mottin, B., Solé, V.A., 2010. Revealing the sfumato Technique of Leonardo da Vinci by X-Ray Fluorescence Spectroscopy. Angew. Chem. Int. Ed. 49, 61256128. https://doi.org/10.1002/anie.201001116

Di Lorenzo, A., Marani, P.C., 2019. Leonardo e la Madonna Litta. Ediz. a colori. Skira, Milan.

Dong, Y., Sørensen, K.M., He, S., Engelsen, S.B., 2017. Gum Arabic authentication and mixture quantification by near infrared spectroscopy. Food Control 78, 144-149. https://doi.org/10.1016/j.foodcont.2017.02.002

Elias, M., Cotte, P., 2008. Multispectral camera and radiative transfer equation used to depict Leonardo's sfumato in Mona Lisa. Appl. Opt. 47, 2146-2154. https://doi.org/10.1364/AO.47.002146

Galli, A., Caccia, M., Bonizzoni, L., Gargano, M., Ludwig, N., Poldi, G., Martini, M., 2020. Deep inside the color: How optical microscopy contributes to the elemental characterization of a painting. Microchem. J. 155, 104730. https://doi.org/10.1016/j.microc.2020.104730

Gargano, M., Bonizzoni, L., Grifoni, E., Melada, J., Guglielmi, V., Bruni, S., Ludwig, N., 2020. Multianalytical investigation of panel, pigments and varnish of The Martyirdom of St. Catherine by Gaudenzio Ferrari (16th century). J. Cult. Herit. https://doi.org/10.1016/j.culher.2020.06.014

Gargano, M., Cavaliere, F., Viganò, D., Galli, A., Ludwig, N., 2017. A new spherical scanning system for infrared reflectography of paintings. Infrared Phys. Technol. 81, 128-136. https://doi.org/10.1016/j.infrared.2016.12.011

Gargano, M., Galli, A., Bonizzoni, L., Alberti, R., Aresi, N., Caccia, M., Castiglioni, I., Interlenghi, M., Salvatore, C., Ludwig, N., Martini, M., 2019. The Giotto's workshop in the XXI century: looking inside the "God the Father with Angels" gable. J. Cult. Herit. 36, 255-263. https://doi.org/10.1016/j.culher.2018.09.016

Gargano, M., Ludwig, N., pandini, davide, 2012. Use of optical fibre in spectrometry and colorimetry with remote probes. J. Int. Colour Assoc. 8.

Gargano, M., Ludwig, N., Poldi, G., 2007. A new methodology for comparing IR reflectographic systems. Infrared Phys. Technol. 49, 249-253. https://doi.org/10.1016/j.infrared.2006.06.013

Gutman Rieppi, N., Price, B.A., Sutherland, K., Lins, A.P., Newman, R., Wang, P., Wang, T., Tague, T.J., 2020. Salvator Mundi: an investigation of the painting's materials and techniques. Herit. Sci. 8, 39. https://doi.org/10.1186/s40494-020-00382-3 
Hark, R.R., Clark, R.J.H., 2010. Raman Microscopy of Diverse Samples of Lapis Lazuli at Multiple Excitation Wavelengths. AIP Conf. Proc. 1267, 315-316.

https://doi.org/10.1063/1.3482531

Hartley, R.I., Gupta, R., 1994. Linear pushbroom cameras, in: Eklundh, J.-O. (Ed.), Computer Vision - ECCV '94, Lecture Notes in Computer Science. Springer, Berlin, Heidelberg, pp. 555-566. https://doi.org/10.1007/3-540-57956-7_63

L. Keith, A. Roy, 1996. Giampietrino, Boltraffio, and the Influence of Leonardo. Natl. Gallery Tech. Bull. 4-19.

L. Keith, A. Roy, R. Morrison, P. Shade, 2011. Leonardo da Vinci's "Virgin of the Rocks": Treatment, Technique and Display. Natl. Gallery Tech. Bull. 32, 32-56.

Legrand, S., Vanmeert, F., Van der Snickt, G., Alfeld, M., De Nolf, W., Dik, J., Janssens, K., 2014. Examination of historical paintings by state-of-the-art hyperspectral imaging methods: from scanning infra-red spectroscopy to computed X-ray laminography. Herit. Sci. 2, 13. https://doi.org/10.1186/2050-7445-2-13

M. Spring, A. Mazzotta, A. Roy, R. Billinge, D. Peggie, 2011. Painting Practice in Milan in the 1490s: The Influence of Leonardo | Technical Bulletin Vol 32 | National Gallery, London. Natl. Gallery Tech. Bull. 32, 78-112.

Miliani, C., Rosi, F., Brunetti, B.G., Sgamellotti, A., 2010. In Situ Noninvasive Study of Artworks: The MOLAB Multitechnique Approach. Acc. Chem. Res. 43, 728-738. https://doi.org/10.1021/ar100010t

Pelagotti, A., Mastio, A.D., Rosa, A.D., Piva, A., 2008. Multispectral imaging of paintings. IEEE Signal Process. Mag. 25, 27-36. https://doi.org/10.1109/MSP.2008.923095

Sarrazin, P., Chiari, G., Gailhanou, M., 2009. A PORTABLE NON-INVASIVE XRD-XRF INSTRUMENT FOR THE STUDY OF ART OBJECTS 12.

Vagnini, M., Miliani, C., Cartechini, L., Rocchi, P., Brunetti, B.G., Sgamellotti, A., 2009. FT-NIR spectroscopy for non-invasive identification of natural polymers and resins in easel paintings. Anal. Bioanal. Chem. 395, 2107-2118. https://doi.org/10.1007/s00216-0093145-6

van der Weerd, J., van Veen, M.K., Heeren, R.M.A., Boon, J.J., 2003. Identification of Pigments in Paint Cross Sections by Reflection Visible Light Imaging Microspectroscopy. Anal. Chem. 75, 716-722. https://doi.org/10.1021/ac020282g

Vandenabeele, P., Verpoort, F., Moens, L., 2001. Non-destructive analysis of paintings using Fourier transform Raman spectroscopy with fibre optics. J. Raman Spectrosc. 32, 263-269. https://doi.org/10.1002/jrs.691

Vandenabeele, P., Wehling, B., Moens, L., Edwards, H., De Reu, M., Van Hooydonk, G., 2000. Analysis with micro-Raman spectroscopy of natural organic binding media and varnishes used in art. Anal. Chim. Acta 407, 261-274. https://doi.org/10.1016/S0003-2670(99)008272 
1

2

\section{SUPPLEMETARY MATERIALS}

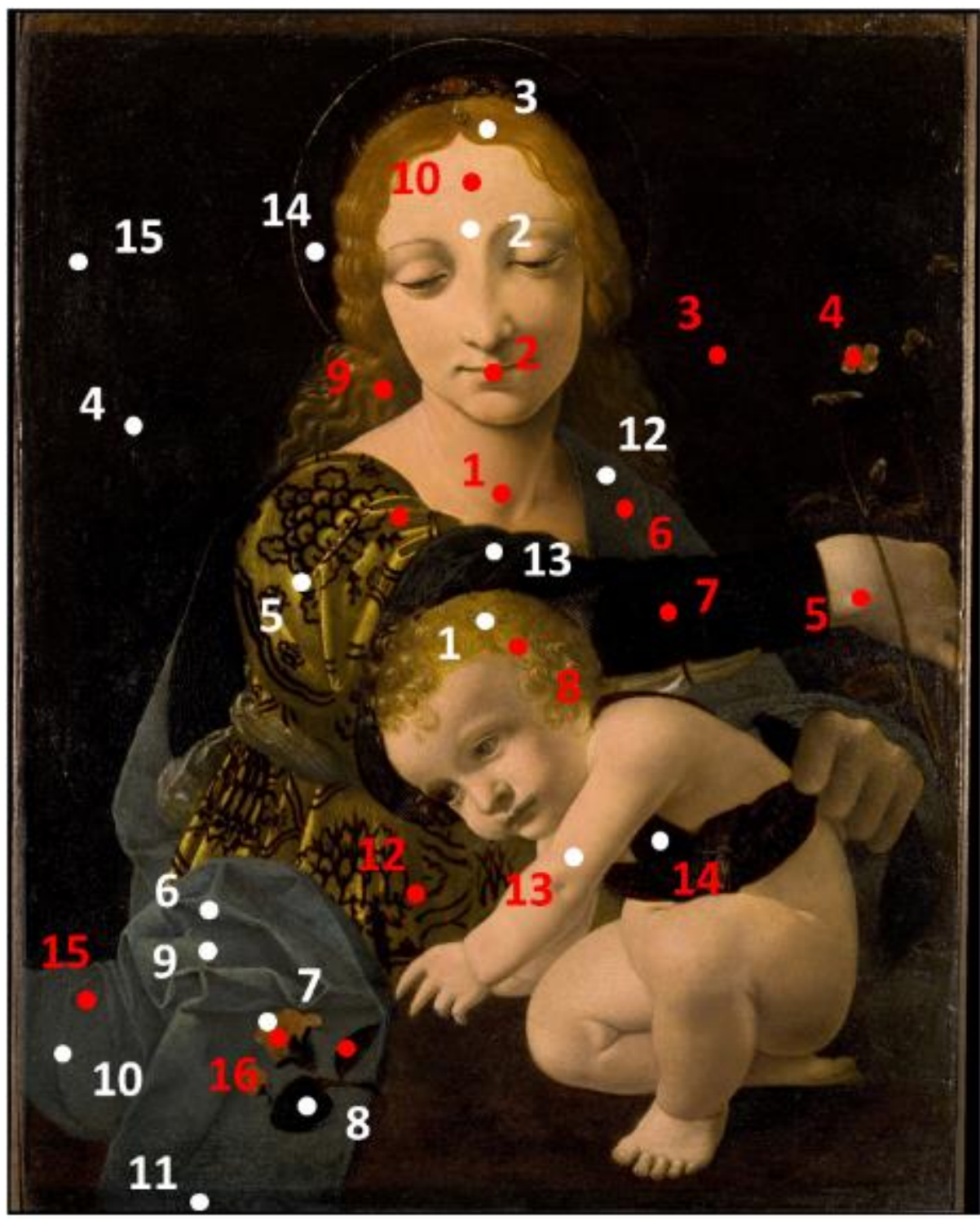

Fig. 1Sa: The Virgin and Child (The Madonna of the Rose) by Giovanni Antonio Boltraffio: red dots indicate XRF, FORS, Raman and OM analysis areas; white dots refer to FT-IR analysis. 


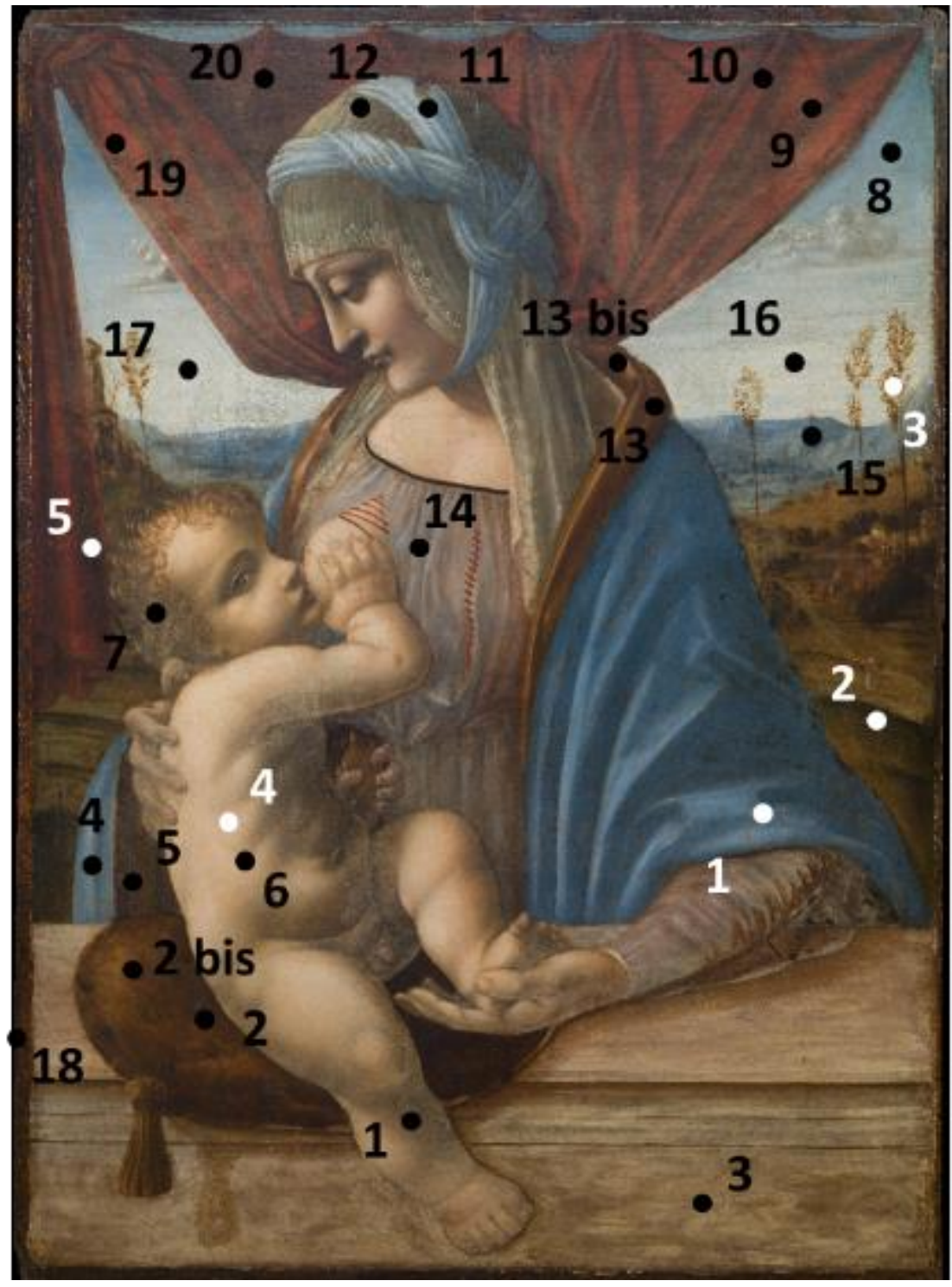

Fig. 1Sb: The Virgin feeding the Child by an unknown painter of the Lombard school: black dots indicate XRF, FORS, Raman and OM analysis areas; white dots refer to FT-IR analysis. 


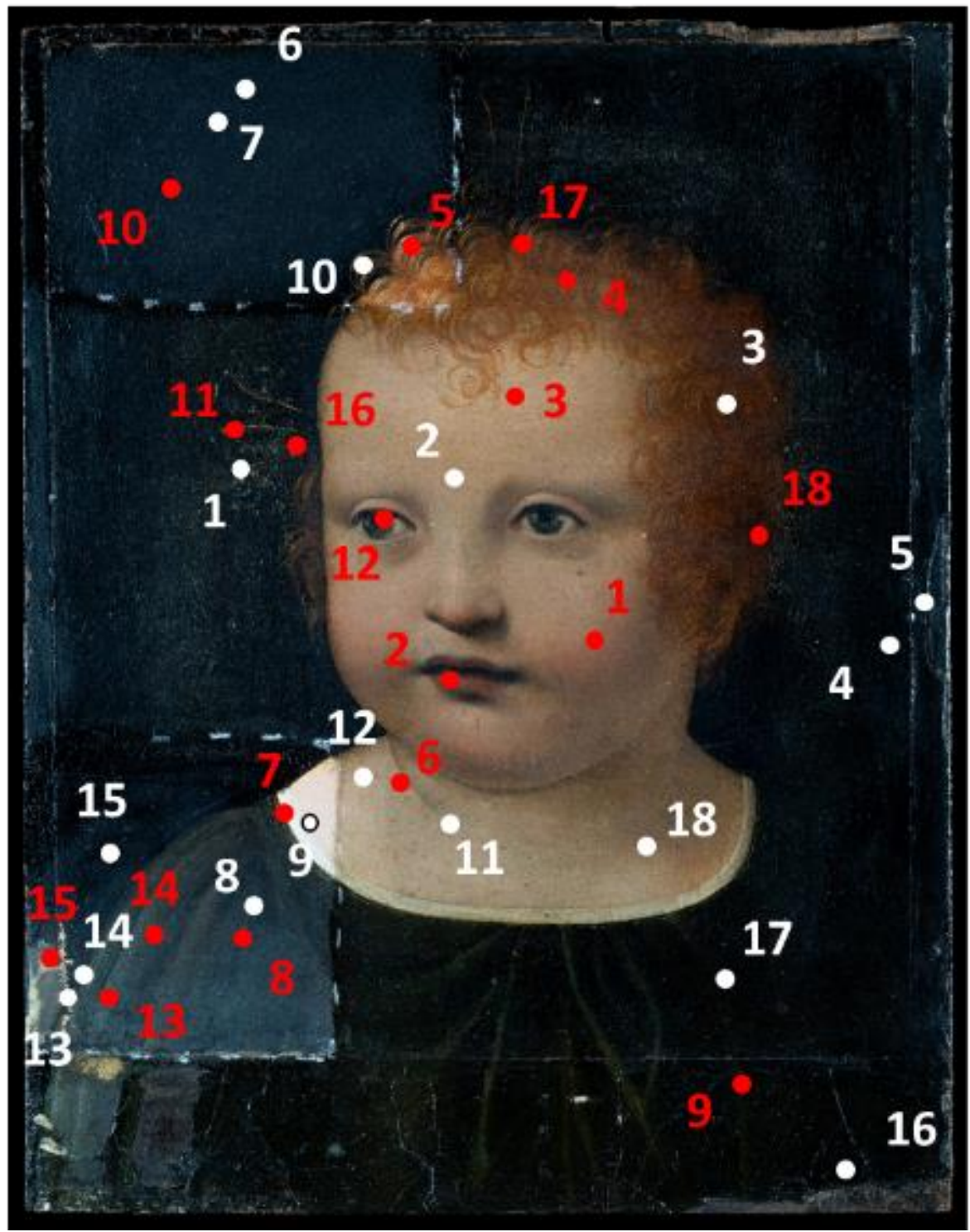

Fig. 1Sc: The Christ Child by Marco d'Oggiono: red dots indicate XRF, FORS, Raman and OM analysis areas; white dots refer to FT-IR analysis. As analyses were performed during restauration intervention, areas of cleaning proof areas are visible. 


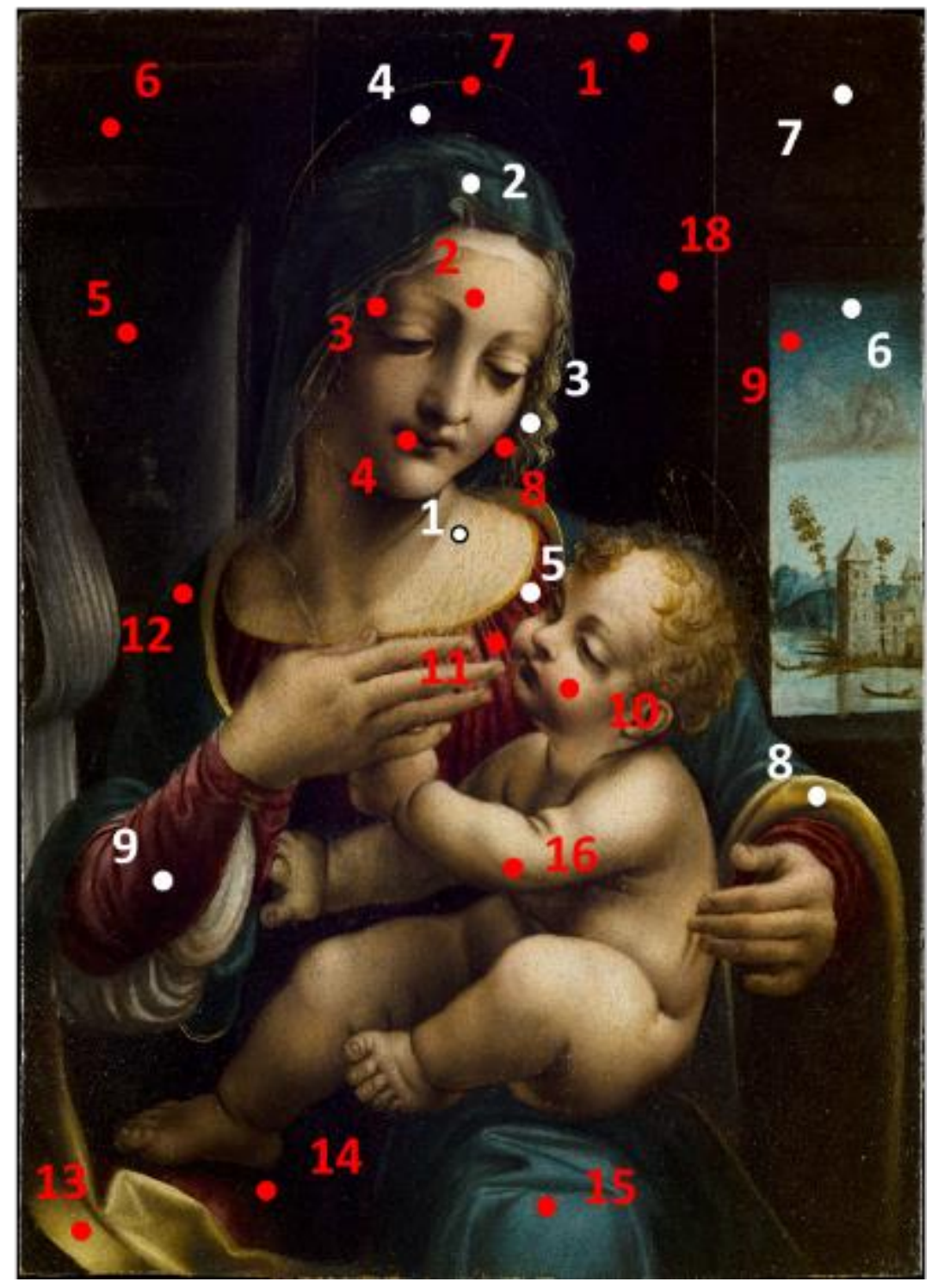

Fig. 1Sd: The Virgin and Child by Francesco Galli: red dots indicate XRF, FORS, Raman and OM analysis areas; white dots refer to FT-IR analysis. 


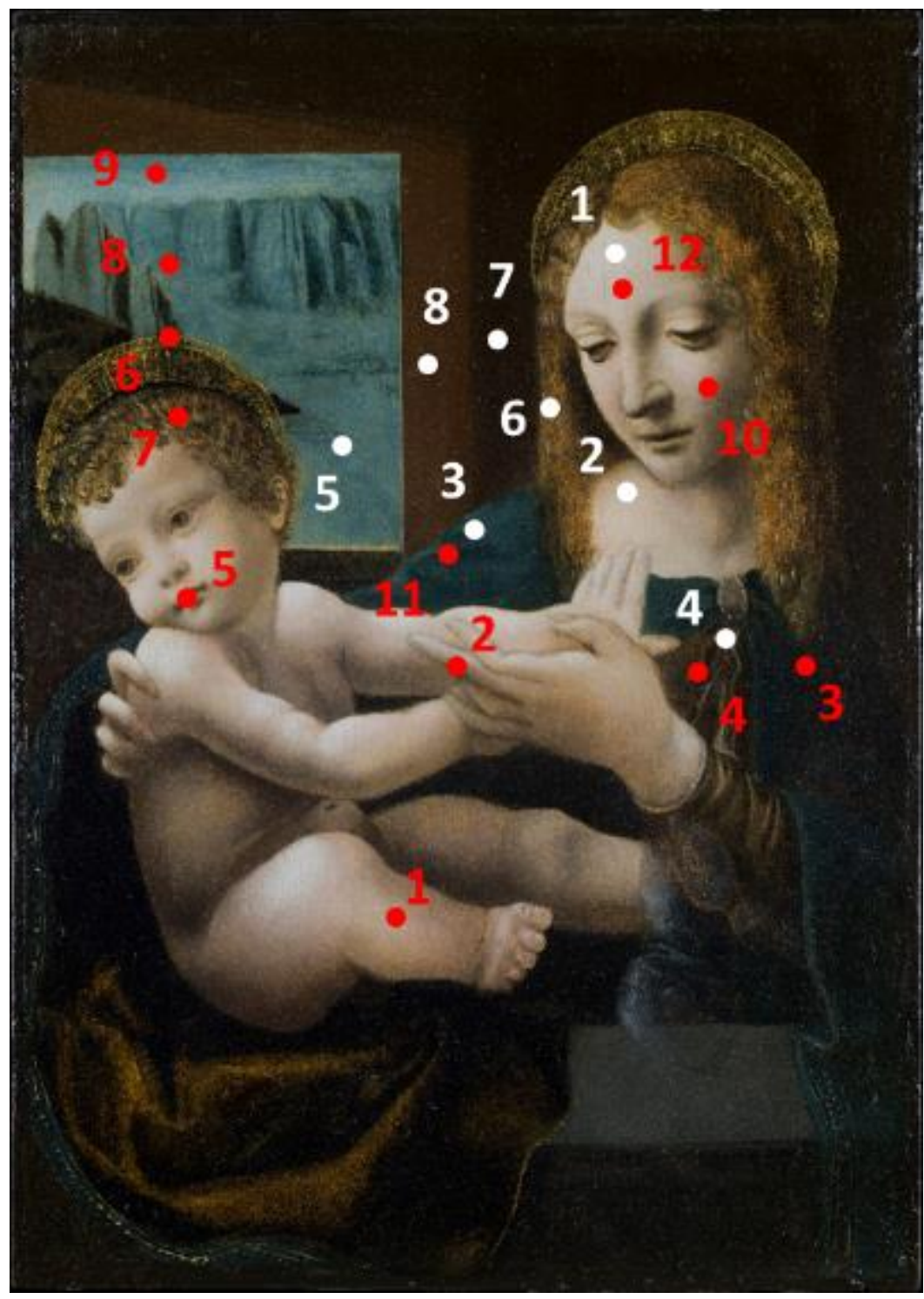

Fig. 1Se: The Virgin and Child by unknown painter of the Lombard school: red dots indicate XRF, FORS, Raman and OM analysis areas; white dots refer to FT-IR analysis. 


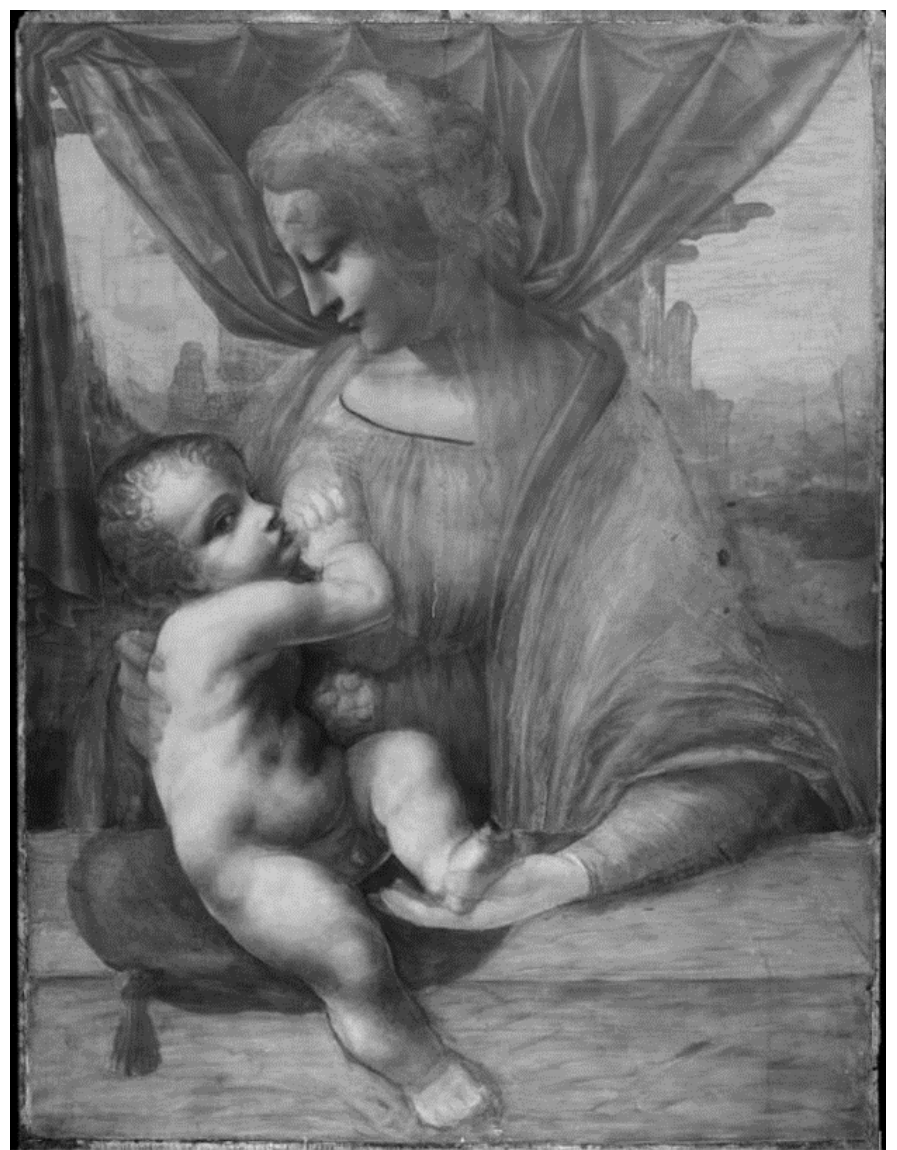

Fig. 2S: Reflectogram 700-1000 nm of The Virgin feeding the Child panel by anonymous

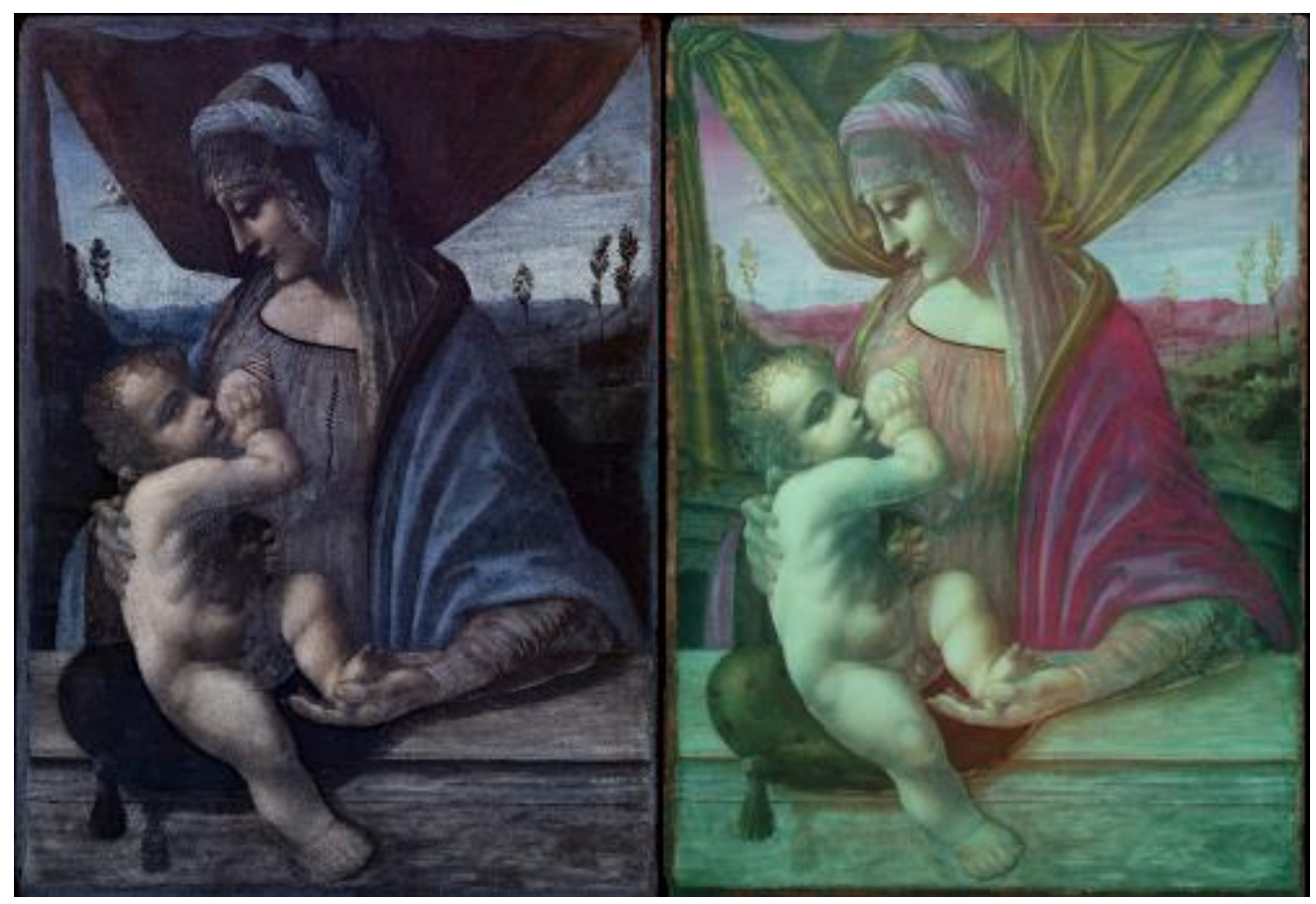

Fig. 3S: UVF and IRFC images for The Virgin feeding the Child panel by the anonymous painter 


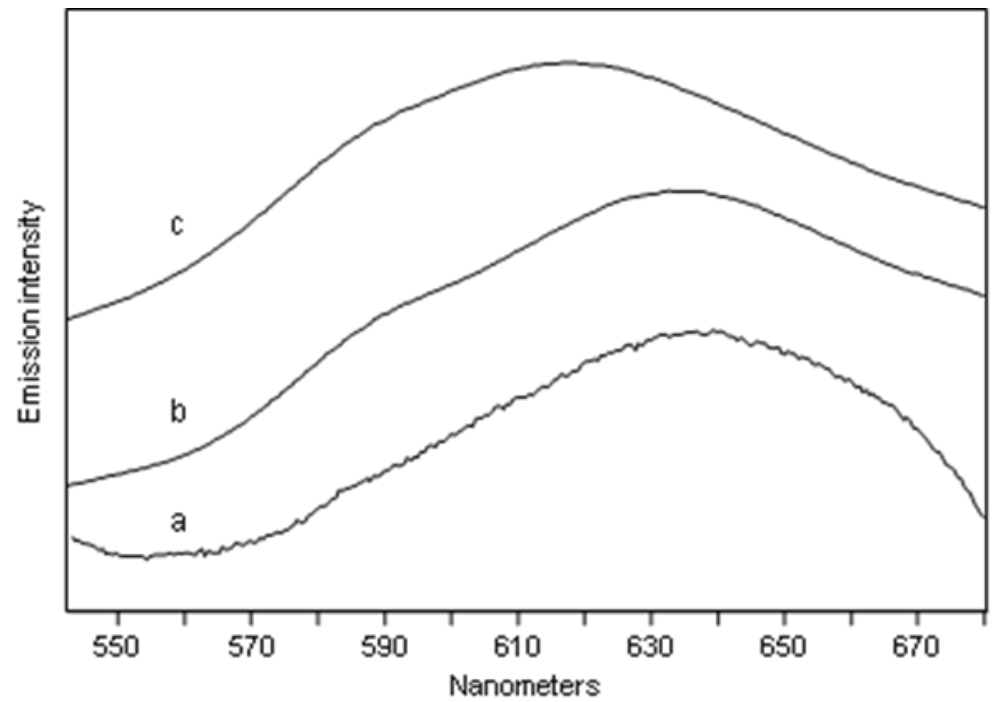

Fig. 4S: Emission spectra ( $\lambda$ exc $435 \mathrm{~nm}$ ) of: (a) detail of the Virgin's red dress in the painting by Francesco Galli; (b) laboratory-made kermes lake spread on canvas with oil binder; (c) laboratory-made madder lake spread on canvas with oil binder. Both lakes were prepared according to traditional recipes, starting respectively from madder roots and kermes dried insects and using $\mathrm{K}_{2} \mathrm{CO}_{3}$ and alum $\mathrm{KAl}(\mathrm{SO} 4) 2 \cdot 12 \mathrm{H}_{2} \mathrm{O}$ as inorganic reagents.

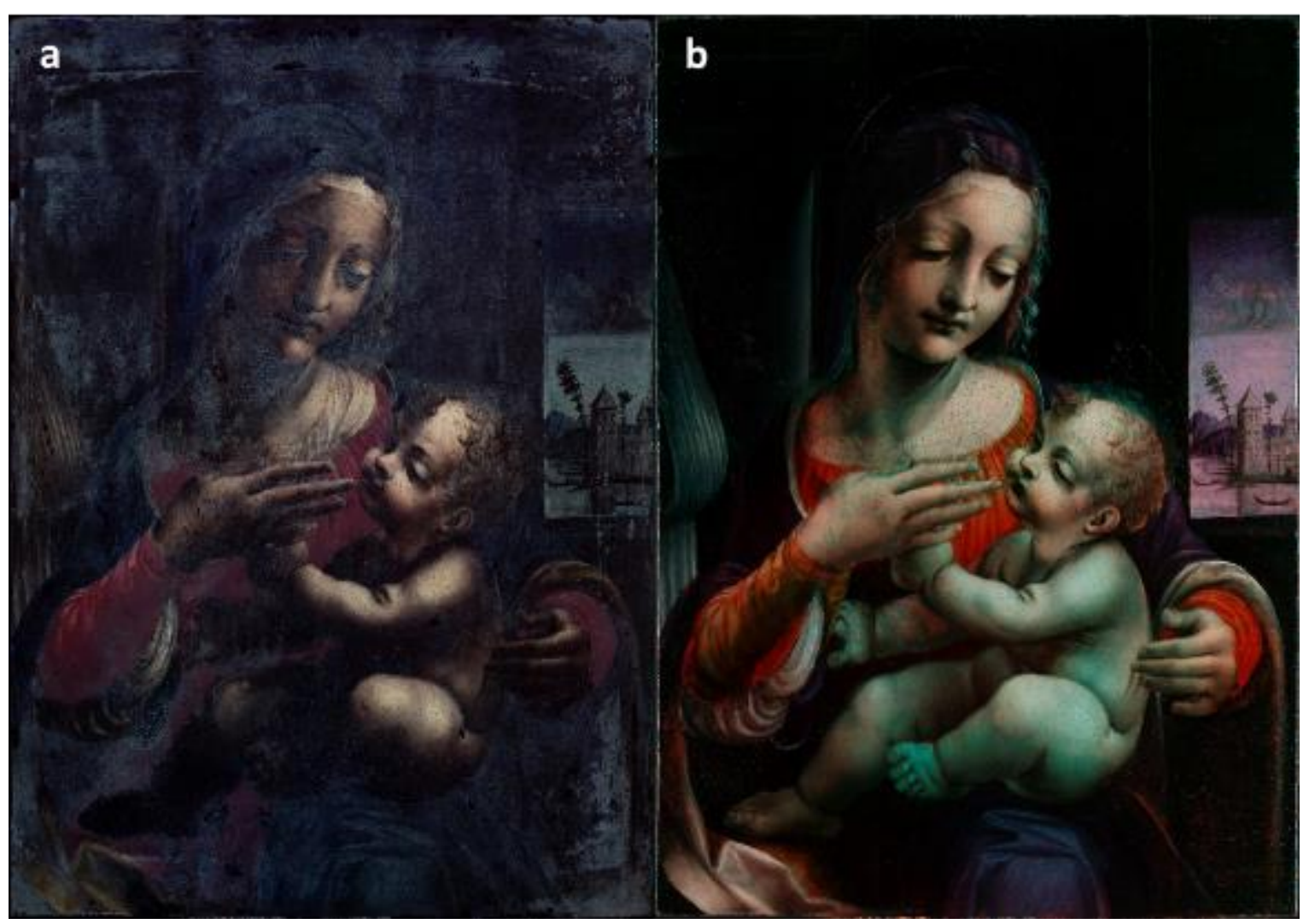

Fig. 5S: UVF (a) and IRFC (b) of Francesco Galli painting. 
1

2

3

4

5

6

7

8

9

10

11

12

13

14

15

16

17

18

19

20

21

22

23

24

25

26

27

28

29

30

31

32

33

34

35

36

37

38

39

40

41

42

43

44

45

46

47

48

49

50

51

52

53

54

55

56

57

58

59

60

61

62

63

64

65

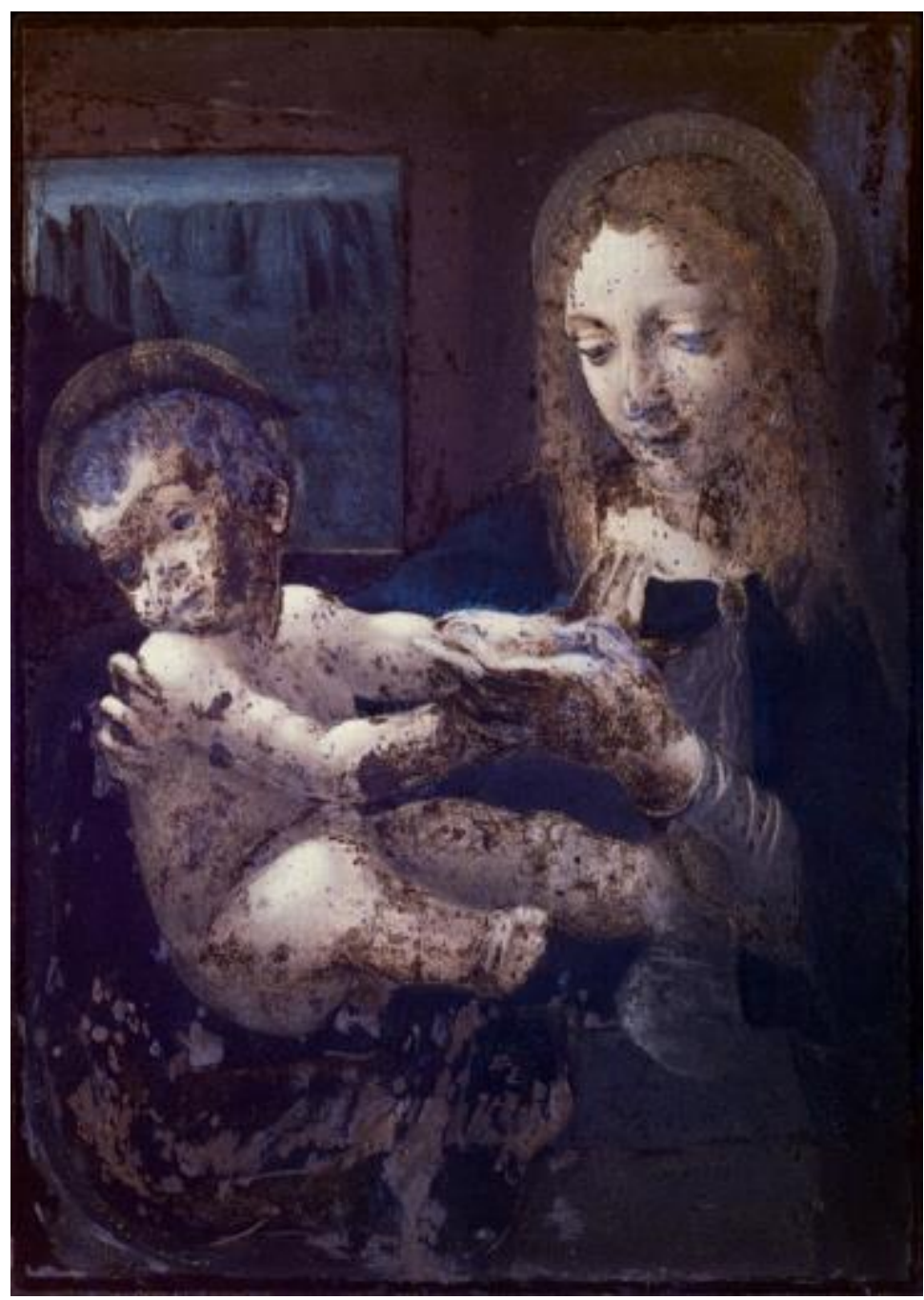

Fig. 6S: UVF of The Virgin and Child by unknown painter of the Lombard school 


\section{Declaration of interests}

$\bigotimes$ The authors declare that they have no known competing financial interests or personal relationships that could have appeared to influence the work reported in this paper.

$\square$ The authors declare the following financial interests/personal relationships which may be considered as potential competing interests:

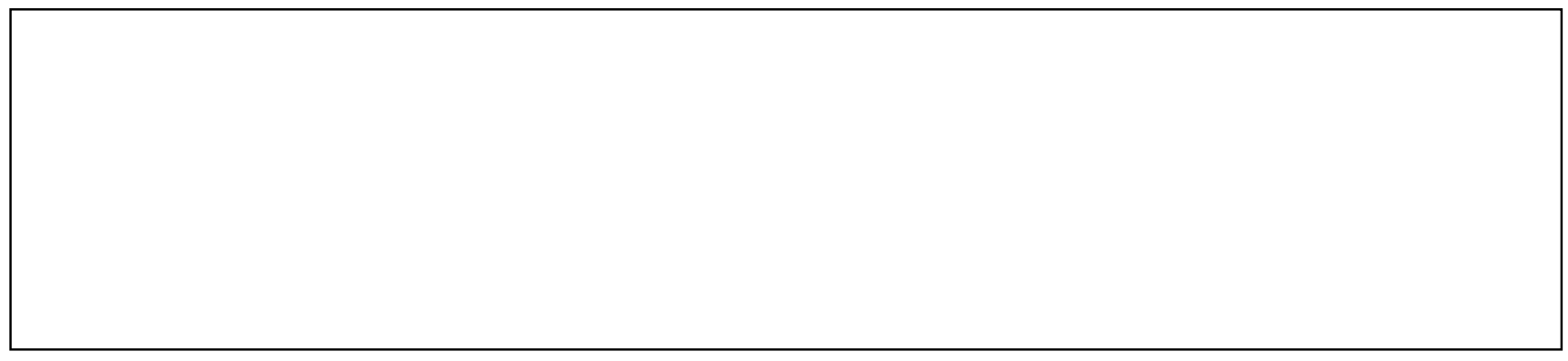

